ESAIM: M2AN 51 (2017) 1903-1929

DOI: $10.1051 / \mathrm{m} 2 \mathrm{an} / 2017011$
ESAIM: Mathematical Modelling and Numerical Analysis

www.esaim-m2an.org

\title{
ANALYTICAL TREATMENT FOR THE ASYMPTOTIC ANALYSIS OF MICROSCOPIC IMPENETRABILITY CONSTRAINTS FOR ATOMISTIC SYSTEMS
}

\author{
Andrea Braides ${ }^{1}$ and Maria Stella Gelli ${ }^{2}$
}

\begin{abstract}
In this paper we provide rigorous statements and proofs for the asymptotic analysis of discrete energies defined on a two-dimensional triangular lattice allowing for fracture in presence of a microscopic impenetrability constraint. As the lattice parameter goes to 0 , we prove that any limit deformation with finite energy is piecewise rigid and we prove a general lower bound with a suitable Griffith-fracture energy density which reflects the anisotropies of the underlying triangular lattice. For such a continuum energy we also provide a class of (piecewise rigid) deformations satisfying "openingcrack" conditions on which the lower bound is sharp. Relying on these results, some consequences have been already presented in the companion paper [9] to validate models in Computational Mechanics in the small-deformation regime.
\end{abstract}

Mathematics Subject Classification. 49J45.

Received October 3, 2016. Revised March 13, 2017. Accepted March 14, 2017.

\section{INTRODUCTION}

In this paper on one hand we analyze continuum models for fracture energies arising from discrete systems of particles linked by interatomic interactions, and on the other hand computational problems deriving from microscopic constraints. The consequences of such an analysis from the standpoint of Computational Mechanics have been described in depth in the paper [9]. Here we provide a rigorous treatment of the related analytical results.

In the simplest model of atomistic interactions, the behavior of a collection of $N$ particles is governed by an energy that can be written as a sum of pair contributions; namely, it can be set in the form

$$
E_{N}\left(\left\{u_{i}\right\}\right)=\sum_{1 \leq i \neq j \leq N} J\left(\left\|u_{i}-u_{j}\right\|\right)
$$

where $u_{i}$ is the position of the $i$ th atom, $\left\|u_{i}-u_{j}\right\|$ the distance between the corresponding pair of atoms, and $J$ is an interatomic potential, which is strongly repulsive at short distances and mildly attractive at long distances.

\footnotetext{
Keywords and phrases. Variational theory of fracture, discrete-to-continuum analysis, $\Gamma$-convergence, Lennard-Jones potentials.

1 Dipartimento di Matematica, Università di Roma Tor Vergata, Roma, Italy.

2 Dipartimento di Matematica, Università di Pisa, Pisa, Italy. gelli@dm.unipi.it
} 
The most common choice for $J$ is the Lennard-Jones potential

$$
J(r)=\frac{c_{1}}{r^{12}}-\frac{c_{2}}{r^{6}}
$$

$\left(c_{1}, c_{2}>0\right)$. Note that in principle the total energy of the system is bounded from below by $N^{2} \min J$; however, a more refined estimate shows that it scales exactly as the number $N$ of atoms. This is in agreement with the intuition that ground states for $E_{N}$ arrange approximately in a regular lattice as $N$ increases (crystallization), so that the energy contribution of each particle is essentially described by the interaction only with its neighbours. At the same time it suggests that models of crystalline solids can be derived directly from such atomic interactions.

The crystallization for Lennard-Jones interactions in the general context described above is still an open problem. An important contribution has been recently given by Theil [24], who has studied a slightly weaker version of crystallization, proving in two dimensions the optimality under compact perturbations of the "minimal" triangular lattice; i.e., the one for whose lattice spacing $\bar{r}$ it is minimal the average energy per particle

$$
e(r)=\sum_{k \in \mathbf{T} \backslash\{\mathbf{0}\}} J(r\|k\|),
$$

which describes the energy of a single particle in the lattice $r \mathbf{T}$ due to the interaction with the other particles, $\mathbf{T}$ being the unit triangular lattice. Once crystallization is achieved, another important issue is whether it is maintained on states other than ground states; i.e., whether to (small) macroscopic deformations there corresponds uniform displacements at the atomic level (Cauchy-Born rule; see Friesecke and Theil [21]).

In order to examine the behavior of atomistic systems far from ground states, under the hypothesis of crystallization we may consider the energy related to a density $\rho$. Noting that $\rho$ is proportional to $r^{-n}$, where $n$ is the space dimension (usually, $n=2,3$ ), this can be expressed as

$$
f(\rho)=\rho\left(C_{1} \rho^{12 / n}-C_{2} \rho^{6 / n}\right),
$$

where $C_{1}, C_{2}$ are determined by computing the energy of a single particle in a uniform lattice (in the case of a triangular lattice $f(\rho)=\rho e(r)$, where $e$ is defined in (1.3) and $r$ is the lattice spacing corresponding to the density $\rho$ ). This function $f$ is non-convex in an interval $\left(0, \rho_{0}\right)$, which highlights a phase transition at low densities, and suggests that large deformations involve a change in the crystalline structure which is instead achieved close to ground states.

From the standpoint of Continuum Mechanics, a description with an hyperelastic bulk energy is expected to hold close to ground states, while the same is expected to fail for a class of large deformations. In the onedimensional case this can be achieved by introducing a fracture energy. Following Truskinovsky [26], given $N$ particle positions $u_{i}$, these can be parameterized with $i=1, \ldots N$ in such a way that $u_{i}>u_{i-1}$, and we may write the energy as

$$
E_{N}\left(\left\{u_{i}\right\}\right)=\sum_{j>i} J\left(u_{j}-u_{i}\right)
$$

As $N$ increases, ground states tend to arrange regularly on a lattice that we may suppose to be $\mathbb{Z}$; i.e., we may suppose that the one-dimensional energy per particle

$$
e(r)=\sum_{k \in \mathbb{N}, k>0} J(r k),
$$

has its minimum at $\bar{r}=1$. In order to introduce a macroscopic deformation gradient, we can now scale and re-parameterize the same particles on $\varepsilon \mathbb{Z}$, where $\varepsilon=\frac{1}{N}$; i.e., $u_{i}=\frac{1}{\varepsilon} u(i \varepsilon)$, so that they all can be seen as discretizations of functions defined on a single interval $[0,1]$. If we scale the energy as

$$
E_{N}\left(\left\{u_{i}\right\}\right)=\sum_{j>i} J\left(\frac{u(j \varepsilon)-u(i \varepsilon)}{\varepsilon}\right) .
$$


then the ground states are discretizations of the identity on $[0,1]$. If we let $N$ increase (or $\varepsilon \rightarrow 0$ ), we may highlight two regimes:

(bulk scaling) under the hypothesis of small perturbations $u(x)=x+\delta v(x)$ with small $\delta$,we have

$$
\frac{u((i+k) \varepsilon)-u(i \varepsilon)}{\varepsilon} \approx k+k \delta v^{\prime}(i \varepsilon)
$$

so that, Taylor expanding $e$ at its minimizer 1 ,

$$
E_{N}\left(\left\{u_{i}\right\}\right)=N \min e+\frac{N}{2} e^{\prime \prime}(1) \delta^{2} \int_{0}^{1}\left|v^{\prime}\right|^{2} \mathrm{~d} x+o\left(\delta^{2}\right) .
$$

(surface scaling) if the macroscopic $u$ is discontinuous at a point parameterized by $i$ then we have an increase from the ground state energy of the order $J(+\infty)-\min e$, which gives a Griffith fracture energy at each such point.

This argument can be made rigorous, and it gives:

- elastic behavior close to ground states, with a linearized description given by the linearization of $J$ at ground states;

- a brittle fracture energy depending on the depth of the well of $J$ with respect to the infinity;

- opening fracture: the possibility of a parameterization with increasing $u$ implies that fracture may only open up, providing a natural non-interpenetration condition;

- surface relaxation: on external boundaries and on internal fracture sites the asymmetry of the atomic arrangements gives an additional surface term, highlighting a microscopic rearrangement close to those surfaces. We note that all the features above can be included in a single analysis by choosing $\delta=N^{-1 / 2}$ in the notation above, so that (up to additive constants) bulk and surface terms have the same scaling. This has been done by Braides, Lew and Ortiz [10]. We also remark that an analysis of local minima and of opening cracks suggests a cohesive fracture energy density of Barenblatt type, which is not directly given by the analyses above. However some cohesive theories can be shown to be "equivalent" to the ones obtained by the limit analysis (see Braides and Truskinovsky $[7,13])$.

The same study for the two or three-dimensional case presents greater difficulties, mainly because a natural parameterization with increasing functions is no longer possible. Hence, some simplifications have been made for this model with the scope of maintaining the features of the complete system of interactions and at the same time allow for an analytical study. A first assumption is to consider displacements as a perturbation from a ground state for which crystallization holds; i.e., in dimension two a perturbation from a state parameterized on the triangular lattice $\mathbf{T}$, or rather on a bounded portion $\Lambda$ of $\mathbf{T}$ (this corresponds to take $N$ as the numbers of points in $\Lambda$ in the previous notation). For such a perturbation it makes sense to assume that only short-range interactions be taken into account. The range of such interaction can be indexed with a subset $S$ of $\mathbf{T} \backslash\{0\}$. The energies replacing $E_{N}$ can be written as

$$
F_{\Lambda}(u)=\sum_{k \in S} \sum_{i, j \in \Lambda, i-j=k} J_{k}\left(\left\|u_{i}-u_{j}\right\|\right),
$$

where $J_{k}$ represents the energy between points whose parameters $i, j$ differ by $k$ in the reference lattice. The simplest choice is considering only nearest-neighbour interactions on $\mathbf{T}$, with $S$ as the unit vectors of the triangular lattice, and $J_{k}$ independent of $k \in S$ a Lennard-Jones potential with minimum in $\bar{r}$. In this simplified model interactions are minimized when the corresponding $u_{i}-u_{j}$ are at distance $\bar{r}$, thus recovering uniform deformations. Unfortunately, such a simplified model admits many additional minimizers, as all deformations which are piecewise homotheties with ratio $\pm \bar{r}$. In fact, if we compose a uniform deformation (e.g. a homothety) of ratio $\bar{r}$ with "folding" along a line of points in $\mathbf{T}$, the resulting nearest neighbours still are at the "minimal" distance $\bar{r}$. 

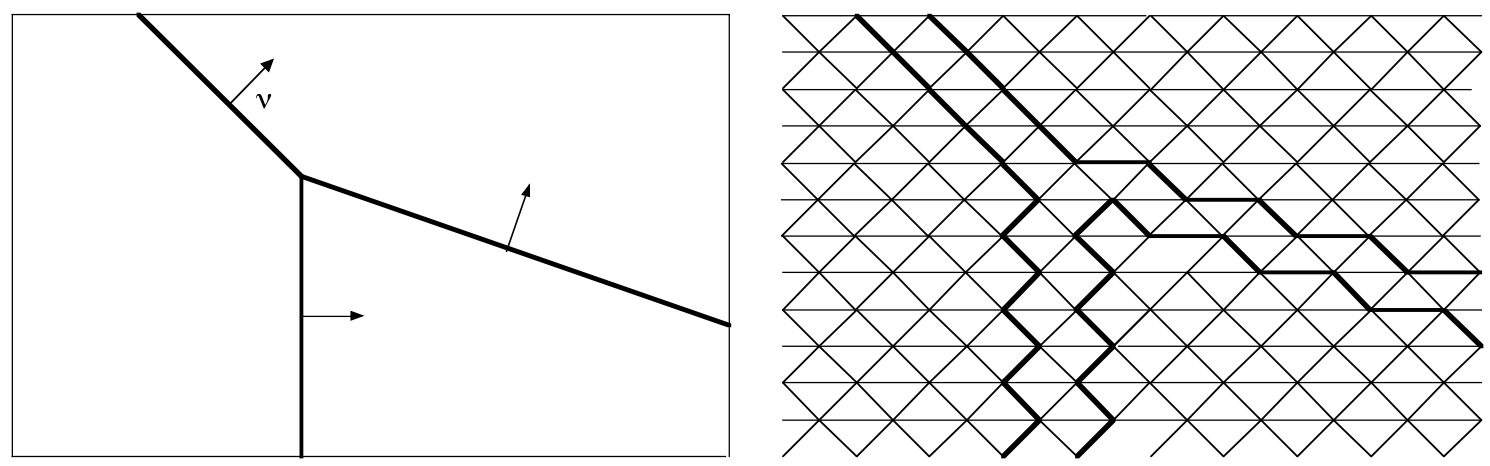

FiguRe 1. A reference configuration with fracture site and its macroscopic normals, and its underlying triangulation.

In order to prevent undesired foldings at a discrete level, Friesecke and Theil [21] proposed to add a three-point condition on neighbouring nodes, corresponding to the usual local non-interpenetration condition in Continuum Mechanics. Indeed, in the case of a triangular lattice, this condition simply amounts to requiring that

$$
\operatorname{det} \nabla u>0 \text {, }
$$

where $u$ is the affine interpolation of the function defined on the vertices of each triangle. In this way only discretizations of homotheties with positive ratio equal to $\bar{r}$ are minimizers of the energy. In their paper, Friesecke and Theil treat the seemingly "un-natural" case of energies parameterized on a square lattice. Actually, when finite-range lattice energies are considered, the choice of the underlying lattice is a matter of convenience, and the square lattice is the simplest where to consider at the same time nearest neighbours and next-to-nearest neighbours to highlight the possibility of non-uniform ground states (failure of the Cauchy-Born rule).

In this paper we treat a two-dimensional system of nearest-neighbour Lennard-Jones interactions with the positive-determinant constraint focusing on the surface scaling. We will use the terminology and techniques of $\Gamma$-convergence applied to a discrete-to-continuum analysis [6]. In this framework we examine the overall behavior of the energies $F_{\Lambda}$ as the size of $\Lambda$ diverges, by considering $\Lambda=\frac{1}{\varepsilon}(\Omega \cap \mathbf{T})$, with $\Omega$ a fixed bounded domain in $\mathbb{R}^{2}$, and using $\Omega \cap \varepsilon \mathbf{T}$ as the set of parameters. The scaled energies we are going to examine will be of the form

$$
F_{\varepsilon}(u)=\sum_{i, j \in \Omega \cap \varepsilon \mathbf{T},|i-j|=\varepsilon} \varepsilon J\left(\left\|\frac{u_{i}-u_{j}}{\varepsilon}\right\|\right),
$$

where $u_{i}$ is the value of the discrete function $u$ at the node $\varepsilon i \in \Omega \cap \varepsilon \mathbf{T}$, and the piecewise-affine interpolation of $u$ on the triangulation related to $\varepsilon \mathbf{T}$ is supposed to satisfy the positive-determinant constraint. The scaling $\varepsilon$ heuristically can be explained by considering, as in the one-dimensional case, the contribution of a set of indices $I$ where $\left\|u_{i}-u_{j}\right\| \gg 1$ and noting that under this scaling the finiteness of the energy asymptotically implies that they have the dimension of a line, so that they can be regarded as an interface. Under these assumption we will address the two issues

- determine whether some condition of "opening crack" still hold in the two-dimensional case;

- characterize a limit continuum surface energy defined on functions with domain $\Omega$.

The other two issues present in the one-dimensional analysis; i.e., the characterization of the bulk energy close to ground states and surface relaxation have been separately addressed in slightly different hypotheses by Braides, Solci and Vitali [12] (for the bulk analysis) and Theil [25] (for the external surface relaxation).

In order to make the analysis clearer we will scale the energies by an additive constant as

$$
F_{\varepsilon}(u)=\sum_{i, j \in \Omega \cap \varepsilon \mathbf{T},|i-j|=\varepsilon} \varepsilon\left(J\left(\left\|\frac{u_{i}-u_{j}}{\varepsilon}\right\|\right)-\min J\right),
$$



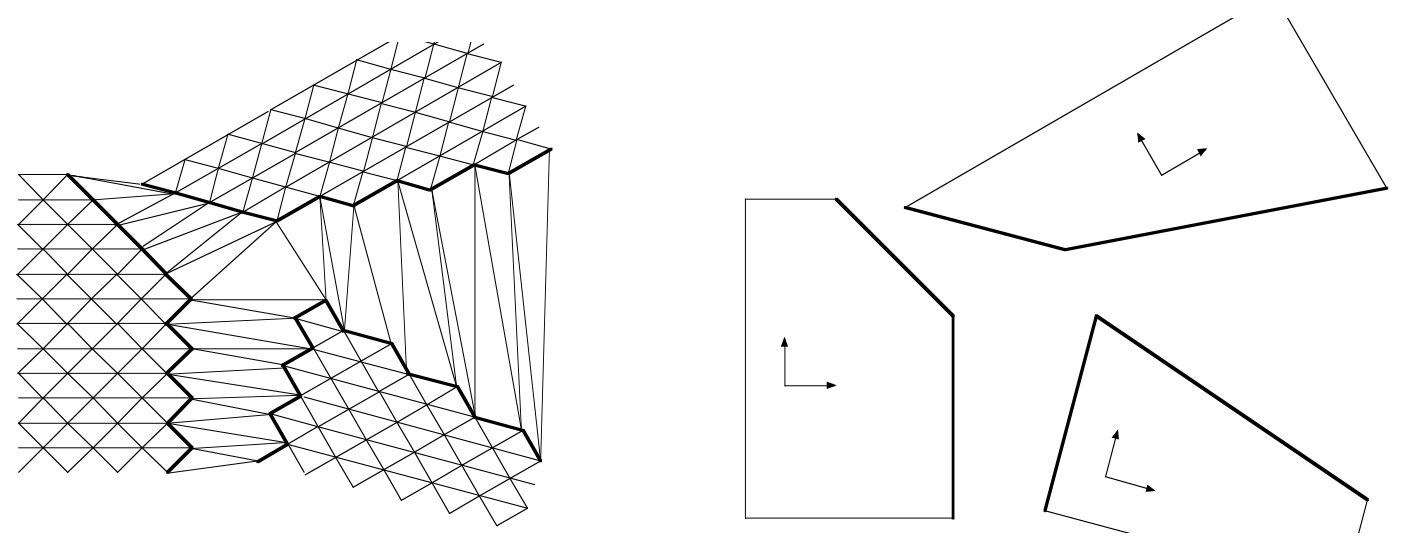

FiguRE 2. A deformed microscopical configuration satisfying the determinant constraint, and its macroscopic limit with the corresponding rotations.

so that the energy density is always positive. As a first remark we will note that, using a result by Chambolle, Giacomini and Ponsiglione [14], gradients of limits of sequences $\left(u_{\varepsilon}\right)$ with equi-bounded energy are piecewise rotations with an underlying partition of $\Omega$ into sets of finite perimeter. This allows us to focus on the boundaries of such sets, where we have a normal $\nu$ on whose two sides we have the values $u^{ \pm}(x)$ of $u$ and two rotations $R^{ \pm}$ among those labeling the sets of the partition. Note that from the standpoint of the microscopical triangulations the interfaces are the limits of triangles of side-length $\varepsilon$ which are deformed by $u_{\varepsilon}$ into triangles with one side (actually two) of diverging length but with the same ordering of the vertices (this corresponds to the positive-determinant constraint). If only one layer of triangles is deformed that gives a limit interface, then the determinant constraint gives a relation between $\nu, u^{ \pm}(x)$ and $R^{ \pm}$. In the simplest case when $\nu$ is orthogonal to one of the unit vectors of $\mathbf{T}$ (we call those vectors coordinate normals), this relation reads as

$$
\left\langle u^{+}(x)-u^{-}(x), R^{ \pm} \nu\right\rangle \geq 0 .
$$

This can be regarded as an opening-fracture condition. If $\nu$ is not a coordinate normal then the openingcrack condition gets more complicated, due to microscopic anisotropies of the lattice, which disfavor cracks not orthogonal to lattice directions.

The analytical description contained in Section 4 provides a lower bound, which is sharp for a class of "opening-crack" configurations (see Sect. 5). This lower bound can be written as a surface energy

$$
\int_{S_{u}} \varphi(\nu) \mathrm{d} \mathcal{H}^{1}
$$

where $S_{u}$ is the fracture set in the reference configuration, and $\varphi$ is an energy density with hexagonal symmetry explicitly computed from $J$. We note that, even if it is not explicit in (1.4), the fracture energy density depends not only on the crack orientation $\nu$ and the crack opening $u^{+}-u^{-}$, but also on the image of the crack in the deformed configuration, described by the tangential derivatives of $u^{ \pm}$on both sides of the crack. Such types of energies seem to be of interest in themselves, and the corresponding analytical techniques still relatively undeveloped (see [3]).

The description in (1.4) is sharp, in the sense that various pathologies may appear when the opening-crack condition is not satisfied. They are described in detail in [9]. Indeed, in that case one must take into account that more than one layer of triangles may be "strongly deformed". This gives a higher energy on the interface, but relaxes the constraints on $\nu, u^{ \pm}(x)$ and $R^{ \pm}$. Moreover, additional energy contributions may be given by points where three or more interfaces meet; in this case, even though the opening-fracture condition above 
may be satisfied on each interface, the system of interfaces may be incompatible with the positive-determinant condition in their common point. It must be observed that introducing a high number of extra interfaces at the discrete level results in a complex non-local form of the final energy.

We finally note that the description of the surface energies suggests the possibility of studying this Lennard-Jones system under the scaling introduced in [10]. Indeed, in the "infinitesimal case" when $u$ is a small variation of a ground state; i.e., $u=i d+\delta v$ with $\delta \ll 1$, then the opening-crack condition reduces to the usual infinitesimal opening-crack condition

$$
\left\langle v^{+}(x)-v^{-}(x), \nu\right\rangle \geq 0
$$

since $R^{ \pm}=i d+o(1)$. This in particular holds when $\delta=\sqrt{\varepsilon}$, which is the scaling that allows both bulk and surface limit energies at the same time, and suggests that in this scaling we obtain a linear elastic energy with a brittle-fracture surface energy subject to the infinitesimal opening-crack condition. The proof of such a result seems of great technical difficulty due to the lack of approximation theorems for functions subject to opening-crack conditions. Related results have been obtained by Friedrich and Schmidt [17-19].

\section{Some NOTATION AND PRELIMINARY RESUlTS}

In the sequel we denote by $\langle\cdot, \cdot\rangle$ the scalar product in $\mathbb{R}^{2}$ and with $|\cdot|$ the usual euclidean norm, both for vectors in $\mathbb{R}^{2}$ and matrices in $\mathcal{M}(2 \times 2 ; \mathbb{R})$, the meaning being clarified by the context. Moreover, in some proofs we will also use the symbol $\langle A, y\rangle$ to denote the duality pair for $A \in \mathcal{M}\left(2 \times 2 ; \mathbb{R}\right.$ ) and $y \in \mathbb{R}^{2}$ (usually simply denoted by $A y)$. For every $x, y \in \mathbb{R}^{2},[x, y]$ denotes the line segment joining $x$ and $y$.

The functional space involved in our discrete-to-continuous analysis will consist of measurable vector-valued functions whose components are special functions with bounded variation. Such functions are commonly used in the variational theories of Fracture (see e.g. the seminal paper by Francfort and Marigo [16], the book by Bourdin, Francfort and Marigo [5] and the review paper [2]).

Definition 2.1. Given $\Omega \subset \mathbb{R}^{2}$ a fixed bounded open set, a function $u \in L^{1}\left(\Omega, \mathbb{R}^{2}\right)$ is a function with bounded variation in $\Omega$, denoted by $u \in B V\left(\Omega, \mathbb{R}^{2}\right)$, if its distributional derivative $D u$ is a vector-valued Radon measure on $\Omega$.

We say that $u$ is a special function with bounded variation in $\Omega$, and we write $u \in S B V\left(\Omega, \mathbb{R}^{2}\right)$, if $u \in$ $B V\left(\Omega, \mathbb{R}^{2}\right)$ and its distributional derivative $D u$ can be represented on any Borel set $A \subset \Omega$ as

$$
D u(A)=\int_{A} \nabla u(x) \mathrm{d} x+\int_{A \cap S_{u}}\left(u^{+}(x)-u^{-}(x)\right) \otimes \nu_{u}(x) \mathrm{d} \mathcal{H}^{1}(x),
$$

for a countably $\mathcal{H}^{1}$-rectifiable set $S_{u}$ in $\Omega$ that coincides $\mathcal{H}^{1}$-almost everywhere with the complement in $\Omega$ of the Lebesgue points of $u$. Moreover, $\nabla u(x)$ is the approximate gradient of $u$ at $x, \nu_{u}(x)$ is a unit normal to $S_{u}$, defined for $\mathcal{H}^{1}$-almost every $x$, and $u^{+}(x), u^{-}(x)$ are the traces of $u$ on both sides of $S_{u}$ (according to the choice of $\left.\nu_{u}(x)\right)$. Here the symbol $\otimes$ stands for the tensorial product of vectors; i.e., for any $a, b \in \mathbb{R}^{2}(a \otimes b)_{i j}:=a_{i} b_{j}$.

For fine properties of $B V$ and $S B V$ functions and a rigorous definition of all the quantities introduced above we refer to [4], Chapter 4. SBV functions enjoy a good compactness property that here is stated in its simplest form, suited for our functionals.

Theorem 2.2. Let $\Omega \subset \mathbb{R}^{2}$ be a bounded open set and let $\left(u_{n}\right) \subset S B V\left(\Omega, \mathbb{R}^{2}\right)$ be satisfying

$$
\sup _{n}\left\{\int_{\Omega}\left|\nabla u_{n}\right|^{p} \mathrm{~d} x+\left\|u_{n}\right\|_{L^{\infty}\left(\Omega, \mathbb{R}^{2}\right)}+\mathcal{H}^{1}\left(S_{u_{n}}\right)\right\}<+\infty,
$$

for some $p>1$. Then there exist a subsequence $u_{n(k)}$ and a function $u \in S B V\left(\Omega, \mathbb{R}^{2}\right)$ such that $u_{n(k)} \rightarrow u$ in measure. Moreover, $\nabla u_{n(k)} \rightarrow \nabla u$ weakly in $L^{p}\left(\Omega, \mathbb{R}^{2}\right)$ and the restrictions of $D u_{n(k)}$ to $S_{u_{n(k)}}$ weakly* converge to the restriction of $D u$ to $S_{u}$. 
As a consequence we easily get that the limit function $u$ in the previous theorem has the further property that $\int_{\Omega}|\nabla u|^{p} \mathrm{~d} x+\|u\|_{L^{\infty}\left(\Omega, \mathbb{R}^{2}\right)}+\mathcal{H}^{1}\left(S_{u}\right)<+\infty$. Moreover estimate (2.1) allows to discuss separately the lower semicontinuity of suitable bulk and surface integral involving, respectively, the absolutely continuous and jump part of the generalized distributional derivative.

In order to treat lower-semicontinuous bulk integrals in the vectorial setting it is necessary to recall the notion of quasiconvexity and quasiconvex envelopes.

Definition 2.3. We say that a lower-semicontinuous integrand $\psi: \mathbb{R}^{2 \times 2} \rightarrow \mathbb{R} \cup\{ \pm \infty\}$ is quasiconvex if for every open set $E$ with positive measure it holds

$$
\psi(A) \leq f_{E} \psi(A+\nabla \varphi(x)) \mathrm{d} x
$$

for every $A \in \mathbb{R}^{2 \times 2}$ and $\varphi \in W_{0}^{1, \infty}\left(E, \mathbb{R}^{2}\right)$. In addition, for any $f: \mathbb{R}^{2 \times 2} \rightarrow \mathbb{R} \cup\{ \pm \infty\}$, the quasiconvex envelope $f^{q c}$ of $f$ is defined as

$$
f^{q c}(A):=\sup \{g(A): g \text { quasiconvex, } g \leq f\} .
$$

If $f$ is real valued and continuous one can give a precise description of its quasiconvex envelope.

Proposition 2.4. Let $f: \mathbb{R}^{2 \times 2} \rightarrow \mathbb{R}$ be continuous. Then

$$
f^{q c}(A)=\inf \left\{f_{E} f(A+\nabla \varphi(x)) \mathrm{d} x: \varphi \in W_{0}^{1, \infty}\left(E, \mathbb{R}^{2}\right)\right\}
$$

for $A \in \mathcal{M}(2 \times 2 ; \mathbb{R})$ and the quantity above is independent of the choice of the bounded open set $E$.

The following theorem deals with lower semicontinuity along sequences of SBV functions weakly converging [4].

Theorem 2.5. Let $\Omega \subset \mathbb{R}^{2}$ be a bounded open set and let $\left(u_{n}\right) \subset S B V\left(\Omega, \mathbb{R}^{2}\right)$ be satisfying

$$
\sup _{n}\left\{\int_{\Omega}\left|\nabla u_{n}\right|^{p} \mathrm{~d} x+\mathcal{H}^{1}\left(S_{u_{n}}\right)\right\}<+\infty
$$

for $p>1$. If $u_{n} \rightarrow u$ in measure, then $u \in S B V\left(\Omega, \mathbb{R}^{2}\right)$. Moreover, for every quasiconvex integrand $\psi: \mathbb{R}^{2 \times 2} \rightarrow$ $[0,+\infty)$ such that

$$
|\psi(X)| \leq C\left(1+|X|^{p}\right)
$$

for every $X \in \mathbb{R}^{2 \times 2}$ and $C$ a positive constant, and for any norm $\varphi$ in $\mathbb{R}^{2}$, there holds

$$
\begin{gathered}
\int_{\Omega} \psi(\nabla u) \mathrm{d} x \leq \liminf _{n} \int_{\Omega} \psi\left(\nabla u_{n}\right) \mathrm{d} x, \\
\int_{S_{u}} \varphi\left(\nu_{u}\right) \mathrm{d} \mathcal{H}^{1} \leq \liminf _{n} \int_{S_{u_{n}}} \varphi\left(\nu_{u_{n}}\right) \mathrm{d} \mathcal{H}^{1} .
\end{gathered}
$$

The final tool we present is a 'slicing result' that allows to treat 2-dimensional energies by reducing to 1-dimensional ones [4].

Before entering into details we introduce some notation. For $\xi \in S^{1}$ let $\Pi^{\xi}:=\left\{y \in \mathbb{R}^{2}:\langle y, \xi\rangle=0\right\}$ be the line through the origin orthogonal to $\xi$. If $y \in \Pi^{\xi}$ and $E \subset \mathbb{R}^{2}$ we set

$$
E^{\xi, y}:=\{t \in \mathbb{R}: y+t \xi \in E\} .
$$

Moreover, if $u: E \rightarrow \mathbb{R}^{2}$ we define the function $u^{\xi, y}: E^{\xi, y} \rightarrow \mathbb{R}^{2}$ by

$$
u^{\xi, y}(t):=u(y+t \xi) .
$$


Theorem 2.6. Let $\Omega \subset \mathbb{R}^{2}$ be a bounded open set and let $u \in S B V\left(\Omega, \mathbb{R}^{2}\right)$. Then, for all $\xi \in S^{1}$ the function $u^{\xi, y}$ belongs to $\operatorname{SBV}\left(\Omega^{\xi, y}, \mathbb{R}^{2}\right)$ for $\mathcal{H}^{1}$-almost every $y \in \Pi^{\xi}$. Moreover for such $y$ we have

$$
\begin{gathered}
\dot{u}^{\xi, y}(t)=\langle\nabla u(y+t \xi), \xi\rangle \quad \text { for almost every } t \in \Omega^{\xi, y}, \\
S\left(u^{\xi, y}\right)=\left\{t \in \mathbb{R}: y+t \xi \in S_{u}\right\}, \\
u^{\xi, y}(t \pm)=u^{ \pm}(y+t \xi) \quad \text { or } \quad u^{\xi, y}(t \pm)=u^{\mp}(y+t \xi),
\end{gathered}
$$

according to the cases $\left\langle\nu_{u}, \xi\right\rangle>0$ or $\left\langle\nu_{u}, \xi\right\rangle<0$ (the case $\left\langle\nu_{u}, \xi\right\rangle=0$ being negligible) and

$$
\int_{\Pi^{\xi}} \sum_{t \in S\left(u^{\xi, y}\right)} g(t) \mathrm{d} \mathcal{H}^{1}(y)=\int_{S_{u}} g(x)\left|\left\langle\nu_{u}, \xi\right\rangle\right| \mathrm{d} \mathcal{H}^{1}
$$

for all Borel functions $g$. Conversely, if $u \in L^{\infty}\left(\Omega, \mathbb{R}^{2}\right)$ and, for all $\xi \in\left\{e_{1}, e_{2}\right\}$ and for $\mathcal{H}^{1}$-almost every $y \in \Pi^{\xi}$, $u^{\xi, y} \in \operatorname{SBV}\left(\Omega^{\xi, y}, \mathbb{R}^{2}\right)$ with

$$
\int_{\Pi^{\xi}}\left(\int_{\Omega^{\xi, y}}\left|\dot{u}^{\xi, y}\right|^{p}+\#\left(S\left(u^{\xi, y}\right)\right)\right) \mathrm{d} \mathcal{H}^{1}(y)<+\infty
$$

then $u \in S B V\left(\Omega, \mathbb{R}^{2}\right)$.

We introduce now a generalization of a rigidity type result in the SBV setting due to Chambolle et al. (see [14]). Before going through the statement we need some preliminary definitions. First we recall the notion of Caccioppoli partition of a domain (see e.g. [15]).

Definition 2.7. We say that a collection of pairwise disjoint measurable sets $\left\{E_{h}\right\}_{h \in \mathbb{N}}$ is a Caccioppoli partition of a bounded open set $\Omega$ if $\cup_{h \in \mathbb{N}} E_{h}=\Omega$ and

$$
\sum_{h \in \mathbb{N}} \operatorname{Per}\left(E_{h}, \Omega\right)<+\infty
$$

Moreover, given any rectifiable set $K \subset \Omega$ we say that a Caccioppoli partition of $\Omega$ is subordinated to $K$ if $\mathcal{H}^{1}\left(\cup_{h \in \mathbb{N}} \partial^{*} E_{h} \backslash K\right)=0$.

The following result is the 2-dimensional version of the one contained in [14].

Theorem 2.8. Let $\Omega \subset \mathbb{R}^{2}$ be a bounded open set and let $u \in S B V\left(\Omega, \mathbb{R}^{2}\right)$ with $\mathcal{H}^{1}\left(S_{u}\right)<+\infty$ and $\nabla u(x) \in$ $\mathcal{S} O(2)$ for almost every $x \in \Omega$. Then there exists a Caccioppoli partition $\left\{E_{h}\right\}_{h \in \mathbb{N}}$ of $\Omega$ subordinated to $S_{u}$ such that for almost every $x \in \Omega$

$$
u(x)=R_{h} x+q_{h} \text { on } E_{h}
$$

where $R_{h} \in \mathcal{S O}(2)$ and $q_{h} \in \mathbb{R}^{2}$.

\section{Formulation of the PROBlem}

In the sequel $\Omega$ will be a bounded open set in $\mathbb{R}^{2}$ with Lipschitz boundary. This regularity assumption is useful to simplify some of the proofs of Section 5 and it can be dropped for the other sections. With fixed discretization step $\varepsilon>0$ the reference lattice is given by $L_{\varepsilon}:=\varepsilon \operatorname{Span}\left(\eta^{1}, \eta^{2} ; \mathbb{Z}^{2}\right)$ where $\eta^{1}=(1,0), \eta^{2}=(1 / 2, \sqrt{3} / 2)$ and $\operatorname{Span}\left(\eta^{1}, \eta^{2} ; \mathbb{Z}^{2}\right)$ denotes the set of all linear combinations of $\eta^{1}, \eta^{2}$ with coefficients in $\mathbb{Z}^{2}$. We introduce also the following notation

$$
\eta^{3}=\eta^{1}-\eta^{2}, \quad \mathrm{~S}=\left\{ \pm \eta^{1}, \pm \eta^{2}, \pm \eta^{3}\right\}
$$

Note that $\mathrm{S}$ is the set of unitary vectors in the lattice $L_{1}$ and for each $i \in L_{1} i+\mathrm{S}$ is the set of its nearest neighbours. 
We define also the set $\mathrm{D}$ of coordinate directions as

$$
\mathrm{D}=\left\{\eta^{\perp}: \eta \in \mathrm{S}\right\}
$$

Dropping the dependence on $\varepsilon$ whenever no confusion may arise, we will use the symbol $\mathrm{T}$ to denote any triangle with vertices in $L_{\varepsilon}$ and sides of length $\varepsilon$ and $\mathcal{T}_{\varepsilon}$ will denote the sets of all such triangles. As already pointed out in the Introduction the choice of the lattice relies on the fact that $L_{1}$ is the simplest Bravais lattice in dimension 2 compatible with a Cauchy-Born hypothesis. Before introducing the precise definition of the functionals object of our analysis we list here some notation used in the following sections for the set of pairs of "nearest-neighbouring" indices $\mathcal{N}_{\varepsilon}$ and for the set of triangles well contained in $\Omega$ :

$$
\begin{gathered}
\mathcal{N}_{\varepsilon}(\Omega)=\left\{(\mathrm{i}, \mathrm{j}) \in L_{\varepsilon} \times L_{\varepsilon}:[\mathrm{i}, \mathrm{j}] \subset \Omega,|\mathrm{i}-\mathrm{j}|=\varepsilon, \mathrm{i} \prec \mathrm{j}\right\}, \\
\mathcal{T}_{\varepsilon}^{c}(\Omega)=\left\{\mathrm{T} \in \mathcal{T}_{\varepsilon}: \mathrm{T} \subset \Omega\right\},
\end{gathered}
$$

where the symbol $i \prec j$ stands for the standard lexicographic order in $\mathbb{R}^{2}$. Thanks to this choice any pair of nearest-neighbouring indices is counted only once in the energy contribution.

With this notation given a discrete vector-valued displacement $u: L_{\varepsilon} \cap \Omega \rightarrow \mathbb{R}^{2}$ we consider its associated energy

$$
\sum_{(i, j) \in \mathcal{N}_{\varepsilon}(\Omega)} \varepsilon J\left(\left|\frac{u(i)-u(j)}{\varepsilon}\right|\right)
$$

where $J:[0,+\infty) \rightarrow[0,+\infty)$ is a continuous function satisfying the following structure properties:

(i) $\min J=J\left(z_{0}\right)=0$;

(ii) $\lim _{z \rightarrow+\infty} J(z)=J_{\infty}>0$;

(iii) $\lim _{z \rightarrow 0+} J(z)=+\infty$;

(iv) for any $\delta>0$ there exits $c_{\delta}>0$ such that $J(z) \geq c_{\delta}\left(z-z_{0}\right)^{2}$ for $\left|z-z_{0}\right| \leq \delta$.

In what follows, for the sake of computational simplicity we assume $z_{0}=1$. Clearly, up to a scaling argument, the analysis remains valid for the general case.

As customary, in order to pass from discrete systems to a continuum formulation, it is convenient to identify a function $\{u(i)\}_{i \in L_{\varepsilon} \cap \Omega} \subset \mathbb{R}^{2}$ with an element of $L^{1}\left(\Omega, \mathbb{R}^{2}\right)$.

Definition 3.1 (Interpolation). Given a discrete vector-valued function $u: L_{\varepsilon} \cap \Omega \rightarrow \mathbb{R}^{2}$ we define its interpolation in the whole $\Omega$ as a function coinciding on each triangle $\mathrm{T} \in \mathcal{T}_{\varepsilon}^{c}(\Omega)$ with the linear interpolation of the values in its vertices.

Note that an interpolation is not uniquely defined on triangles close to $\partial \Omega$. This will not affect our arguments since convergences are always considered in the interior of $\Omega$. We will identify a discrete function with its interpolation and maintain the same notation for both the discrete and continuous version, the notation being clarified by the context.

Remark 3.2. In this setting another common procedure is to identify a discrete function with a piecewiseconstant element in $L^{1}\left(\Omega, \mathbb{R}^{2}\right)$ by assigning constant value $u(\mathbf{i})$ on the cell $\left\{\mathbf{i}+\varepsilon\left(\lambda \eta^{1}+\mu \eta^{2}\right): \lambda, \mu \in[0,1]\right\}$, $i \in L_{\varepsilon} \cap \Omega$. In fact, none of the results stated in the following would be affected by this choice (see the discussion contained in [1]). Considering discrete functions as continuous piecewise-affine ones allows us to formulate the orientation-preserving constraint in terms of the standard determinant of $\nabla u$.

We are now able to introduce the class of admissible functions; i.e., a proper class of vector-valued functions on the lattice $L_{\varepsilon}$ that are orientation preserving:

$$
\mathcal{A} d_{\varepsilon}(\Omega)=\left\{u: L_{\varepsilon} \cap \Omega \rightarrow \mathbb{R}^{2}: \operatorname{det}(\nabla u)>0 \text { a.e. in } \mathcal{T}_{\varepsilon}^{c}(\Omega)\right\} .
$$


Finally, we define the functional $E_{\varepsilon}$ on $L^{1}\left(\Omega, \mathbb{R}^{2}\right)$ as

$$
E_{\varepsilon}(u)= \begin{cases}\sum_{\substack{(i, j) \in \mathcal{N}_{\varepsilon}(\Omega) \\+\infty}} \varepsilon\left(\left|\frac{u(\mathrm{i})-u(\mathrm{j})}{\varepsilon}\right|\right) & \text { if } \quad u \in \mathcal{A} d_{\varepsilon}(\Omega) \\ & \text { if } \quad u \in L^{1}\left(\Omega, \mathbb{R}^{2}\right) \backslash \mathcal{A} d_{\varepsilon}(\Omega) .\end{cases}
$$

Remark 3.3. Note that energies defined in (3.1) account only for interactions well contained in $\Omega$ and the orientation-preserving constraint is not imposed a priori on an affine extension of $\mathrm{u}$ on the triangles intersecting $\partial \Omega$. Actually, if one is interested in minimum problems endowed with boundary conditions (together with some perturbation or fidelity terms), a standard procedure in the framework of $\Gamma$-convergence is to focus on the 'principal' part of the total energy, neglecting at first boundary data. Once this task is accomplished one can further deal with the initial problems up to modifying the limit energy according to the contribution arising from recovery sequences with correct boundary datum.

To proceed further with our analysis we need to fix a convergence on $L^{1}\left(\Omega, \mathbb{R}^{2}\right)$.

Definition 3.4 (Discrete-to-continuum convergence). According to our identification of discrete functions with interpolations, given $u_{\varepsilon}, u \in L^{1}\left(\Omega, \mathbb{R}^{2}\right)$ we say that $u_{\varepsilon} \rightarrow u$, and we simply write $u_{\varepsilon} \rightarrow u$, if we have $\sup _{\varepsilon}\left\|u_{\varepsilon}\right\|_{L^{\infty}\left(\Omega, \mathbb{R}^{2}\right)}<+\infty$ and $\lim _{\varepsilon \rightarrow 0^{+}}\left\|u_{\varepsilon}-u\right\|_{L^{1}\left(\Omega, \mathbb{R}^{2}\right)}=0$.

\section{Compactness And A LOWER BOUnd}

In this section we first provide a description of the domain of any $\Gamma$-limit functional of the discrete energies defined in (3.1). As already mentioned in the Introduction, this space consists of piecewise rigid deformations $u$ with finite crack energy in the sense of Griffith's theory; i.e., $\mathcal{H}^{1}\left(S_{u}\right)<+\infty$.

As a second result we exploit geometric measure theory techniques to establish a lower-bound estimate for the $\Gamma$-liminf $E_{\varepsilon} E_{\varepsilon}(u)$ without imposing any a priori hypothesis on the deformation $u$. Note that in the next section this bound will be proved to be optimal for the class of piecewise rigid deformations "with opening fracture" in the sense of the anisotropies of the reference lattice. For such deformations the limit fracture energy is simply governed by an anisotropic Griffith-type energy density which reflects the anisotropies of the underlying triangular lattice.

Proposition 4.1. Let $\left\{u_{\varepsilon}\right\} \subset \mathcal{A} d_{\varepsilon}(\Omega)$ be such that $\liminf E_{\varepsilon}\left(u_{\varepsilon}\right)<+\infty$ and

$$
\sup _{\varepsilon}\left\|u_{\varepsilon}\right\|_{L^{\infty}\left(\Omega, \mathbb{R}^{2}\right)}<+\infty .
$$

Then there exists a Borel function $u$ such that, up to subsequences, $u_{\varepsilon} \rightarrow u$ in $L^{1}\left(\Omega, \mathbb{R}^{2}\right)$. Moreover,

(i) (finite Griffith fracture energy) $u \in S B V\left(\Omega, \mathbb{R}^{2}\right)$ with $\mathcal{H}^{1}\left(S_{u}\right)<+\infty$;

(ii) (piecewise rigidity) there exists a Caccioppoli partition $\left\{E_{h}\right\}_{h \in \mathbb{N}}$ subordinated to $S_{u}$ such that for almost every $x \in E_{h}$ we have $u(x)=R_{h} x+q_{h}$ for suitable $R_{h} \in \mathcal{S} O(2)$ and $q_{h} \in \mathbb{R}^{2}$.

Proof. As a first step we observe that any pair $(i, j) \in \mathcal{N}_{\varepsilon}(\Omega)$ belongs to two different triangles having the line segment $[i, j]$ as a side. Hence if we take into account a factor $1 / 2$ we may estimate the energies $E_{\varepsilon}(u)$ from below with the integral functionals obtained as a superposition of gradient energies indexed by the triangles $T$ varying in $\mathcal{T}_{\varepsilon}^{c}(\Omega)$. Actually, for any $B$ open set compactly supported in $\Omega$ and for any $v \in \mathcal{A} d_{\varepsilon}(\Omega)$, for $\varepsilon$ small enough we have

$$
\begin{aligned}
E_{\varepsilon}(v) & \geq \frac{\varepsilon}{2|T|} \sum_{T \in \mathcal{T}_{\varepsilon}^{c}(\Omega)} \sum_{k=1}^{3} \int_{T} J\left(\left|\left\langle\nabla v, \eta^{k}\right\rangle\right|\right) \mathrm{d} x \\
& \geq \frac{2}{\sqrt{3} \varepsilon} \int_{B} \sum_{k=1}^{3} J\left(\left|\left\langle\nabla v, \eta^{k}\right\rangle\right|\right) \mathrm{d} x .
\end{aligned}
$$


According to this standpoint, we are led to considering integral functionals with energy density $\hat{J}(A):=$ $\sum_{k=1}^{3} J\left(\left|\left\langle A, \eta^{k}\right\rangle\right|\right)$. Hence, with fixed any $s \in(0,1)$, we distinguish "good" or "bad" triangles $T$ depending whether the triangle energy overcomes the threshold $s J_{\infty}$ or not. Using a standard separation of scales (see [8]) we set

$$
\mathcal{I}_{\varepsilon}=\left\{T \in \mathcal{T}_{\varepsilon}^{c}(\Omega): \sum_{k=1}^{3} J\left(\left|\left\langle\nabla u_{\varepsilon}, \eta^{k}\right\rangle\right|\right)>s J_{\infty}\right\},
$$

and define $v_{\varepsilon} \in S B V\left(\Omega, \mathbb{R}^{2}\right)$ on any $T \in \mathcal{T}_{\varepsilon}$ such that $T \cap \Omega \neq \emptyset$ as

$$
v_{\varepsilon}=\left\{\begin{array}{lll}
u_{\varepsilon} & \text { if } & T \in \mathcal{T}_{\varepsilon} \backslash \mathcal{I}_{\varepsilon} \\
I & \text { if } & T \in \mathcal{I}_{\varepsilon},
\end{array}\right.
$$

with $I$ denoting the identity deformation. By construction the sequence $\left(v_{\varepsilon}\right)$ lies in $S B V\left(\Omega, \mathbb{R}^{2}\right)$ and for any $\varepsilon>0$ its jump set $S_{v_{\varepsilon}}$ is contained in the boundary of the union of the triangles in $\mathcal{I}_{\varepsilon}$.

Moreover, for any open set $B$ compactly supported in $\Omega$ inequality (4.2) can be rewritten in terms of $v_{\varepsilon}$ as

$$
E_{\varepsilon}\left(u_{\varepsilon}\right) \geq \frac{2}{\sqrt{3} \varepsilon} \int_{B} \hat{J}\left(\nabla v_{\varepsilon}\right) \mathrm{d} x+c s J_{\infty} \varepsilon \#\left(\mathcal{I}_{\varepsilon}\right)
$$

for a positive constant $c$. This implies at once that the functions $u_{\varepsilon}$ and $v_{\varepsilon}$ differ in a set of vanishing Lebesgue measure so that it is enough to prove that $v_{\varepsilon}$ is compact in $S B V\left(\Omega, \mathbb{R}^{2}\right)$.

Thanks to hypothesis (iii) on $J$, the set

$$
\mathcal{K}=\left\{A \in \mathcal{M}(2 \times 2 ; \mathbb{R}): \hat{J}(A) \leq s J_{\infty}\right\}
$$

is a compact set in $\mathcal{M}(2 \times 2 ; \mathbb{R})$, and this easily provides the estimate

$$
\sup _{\varepsilon}\left\|\nabla v_{\varepsilon}\right\|_{L^{2}\left(B, \mathbb{R}^{2}\right)}<+\infty .
$$

On the other hand, hypothesis (iv) ensures that there exists a constant $c=c(s)$ such that (4.4) can be further sharpened as

$$
E_{\varepsilon}\left(u_{\varepsilon}\right) \geq \frac{1}{\varepsilon} \int_{B} \sum_{k=1}^{3} c(s)\left(\left|\left\langle\nabla v_{\varepsilon}, \eta^{k}\right\rangle\right|-1\right)^{2} \mathrm{~d} x+c s J_{\infty} \mathcal{H}^{1}\left(S_{v_{\varepsilon}} \cap B\right) .
$$

By Lemma 4.3 we can find a positive $c$ such that for any $A \in \mathcal{K}$ there exists $R=R(A) \in \mathcal{S O}(2)$ with $\sum_{k=1}^{3}\left(\left|\left\langle A, \eta^{k}\right\rangle\right|-1\right)^{2} \geq c|A-R|^{2}=c \operatorname{dist}^{2}(A, \mathcal{S} O(2))$, thus

$$
E_{\varepsilon}\left(u_{\varepsilon}\right) \geq \frac{c(s)}{\varepsilon} \int_{B} \operatorname{dist}^{2}\left(\nabla v_{\varepsilon}, \mathcal{S} O(2)\right) \mathrm{d} x+c s J_{\infty} \mathcal{H}^{1}\left(S_{v_{\varepsilon}} \cap B\right) .
$$

Taking into account hypothesis (4.1) a straightforward application of Theorem 2.2, and the $L^{1}$ convergence to 0 of $v_{\varepsilon}-u_{\varepsilon}$, yields that there exists $u \in S B V\left(\Omega, \mathbb{R}^{2}\right)$ with $\mathcal{H}^{1}\left(S_{u}\right)<+\infty$ such that $u_{\varepsilon} \rightarrow u$ in $L^{1}\left(\Omega, \mathbb{R}^{2}\right)$.

To prove (ii) we take advantage of a relaxation argument together with some rigidity estimates. Indeed, as a first step we prove that $\nabla u(x) \in \mathcal{S} O(2)$ for almost every $x \in \Omega$. To this end denote $\psi(A):=\operatorname{dist}^{2}(A, \mathcal{S} O(2))$ and estimate the right-hand side of (4.6) as

$$
\varepsilon E_{\varepsilon}\left(u_{\varepsilon}\right) \geq c(s) \int_{B} \psi^{q c}\left(\nabla v_{\varepsilon}\right) \mathrm{d} x+c \varepsilon s J_{\infty} \mathcal{H}^{1}\left(S_{v_{\varepsilon}} \cap B\right) .
$$

Passing to the liminf and using (2.4) we get at once that

$$
\int_{B} \psi^{q c}(\nabla u) \mathrm{d} x=0 .
$$


Hence $\psi^{q c}(\nabla u(x))=0$ almost everywhere in $\Omega$ and by applying Lemma 4.4 with $A=\nabla u(x)$ we also get that the approximate gradient $\nabla u$ is a rotation for almost every $x \in B$. Once this fact is established, (ii) follows by applying Theorem 2.8 recursively to $u$ with $\Omega$ replaced by any open set $B$ compactly supported in $\Omega$ and then letting $B$ invading $\Omega$.

Remark 4.2. Hypothesis (4.1) is essential to deduce a compactness result for sequences equibounded in energy and avoids the un-physical situation of particles escaping to infinity (modelled by arbitrarily large translations).

The following lemmas complete the proof of Proposition 4.1. The first one provides an estimate from above on the function $\psi(A):=\operatorname{dist}^{2}(A, \mathcal{S O}(2)), A \in \mathcal{M}(2 \times 2, \mathbb{R})$ (to be used in (4.6)), the second one computes the zero set of its quasiconvex envelope. Note that the this kind of results are widely used in variational problems involving crystal microstructures.

Lemma 4.3. There exists a positive constant $c$ such that for any $A \in \mathcal{M}(2 \times 2 ; \mathbb{R})$ with $\operatorname{det} A>0$ we have that

$$
\sum_{k=1}^{3}\left(\left|\left\langle A, \eta^{k}\right\rangle\right|-1\right)^{2} \geq c \operatorname{dist}^{2}(A, \mathcal{S O}(2)),
$$

where $\eta^{1}=(1,0), \eta^{2}=(1 / 2, \sqrt{3} / 2)$ and $\eta^{3}=\eta^{1}-\eta^{2}$.

Proof. Let $A \in \mathcal{M}(2 \times 2 ; \mathbb{R})$ with $\operatorname{det} A>0$ be fixed; then $A$ admits a polar decomposition as $A=R U$ with $R \in \mathcal{S O}(2)$ and $U$ a positive-definite matrix that coincides with the square root of the symmetric matrix $A^{t} A$ (see for instance [23]). Since (4.8) is invariant under left composition with rotations, it is enough to prove that

$$
\sum_{k=1}^{3}\left(\left|\left\langle U, \eta^{k}\right\rangle\right|-1\right)^{2} \geq c|U-I|^{2}
$$

for $U \in \mathcal{M}(2 \times 2 ; \mathbb{R})$ strictly positive-definite matrix.

First for the sake of clarity we treat the case in which $U$ is diagonal and we denote its eigenvalues as $1+x, 1+y$ with $x, y \in(-1,+\infty)$. Let $g$ be defined as

$$
g(x, y)=x^{2}+2\left(\sqrt{\frac{(1+x)^{2}}{4}+\frac{3(1+y)^{2}}{4}}-1\right)^{2}
$$

then (4.9) rewrites as

$$
g(x, y) \geq c\left(x^{2}+y^{2}\right)
$$

for some positive $c$ independent of $x, y$ with $(x, y) \in(-1,+\infty) \times(-1,+\infty)$. Arguing by continuity, the inequality above holds true if and only if it holds for $x, y$ with $(x, y) \in[-1,+\infty) \times[-1,+\infty)$. Taking into account that $g(x, y)$ and $x^{2}+y^{2}$ have the same order for $|(x, y)| \rightarrow+\infty$ and that $g(x, y), x^{2}+y^{2}$ vanish only at $(0,0)$, we may confine to prove the inequality above in a neighbourhood of the origin $(0,0)$. To this end consider the following Taylor expansion of $g$ :

$$
\begin{aligned}
g(x, y) & =x^{2}+2\left(\sqrt{1+\frac{x+3 y}{2}+\frac{x^{2}+3 y^{2}}{4}}-1\right)^{2} \\
& =x^{2}+2\left(\frac{x+3 y}{4}+\frac{x^{2}+3 y^{2}}{8}+o(x+3 y)\right)^{2} \\
& =x^{2}+\frac{x^{2}+6 y x+9 y^{2}}{8}+o\left(x^{2}+y^{2}\right) \\
& =\frac{9}{8}\left(x^{2}+y^{2}\right)+\frac{3}{4} x y+o\left(x^{2}+y^{2}\right)
\end{aligned}
$$


and use the inequality $2 x y \geq-x^{2}-y^{2}$ to get

$$
g(x, y)=\left(x^{2}+y^{2}\right)\left(\frac{3}{4}+o(1)\right)
$$

We finally deduce that for $|(x, y)|$ small enough one has $g(x, y) \geq \frac{1}{3}\left(x^{2}+y^{2}\right)$ and conclude the proof for diagonal matrices.

For a general positive-definite matrix $u$, taking into account the classical diagonalization results in the Jordan decomposition theory (see [23]), there exists $R \in \mathcal{S} O(2)$ such that $R^{t} U R$ is diagonal. Hence we are left to prove the inequality above, that is,

$$
\sum_{k=1}^{3}\left(\left|\left\langle D, \tilde{\eta}^{k}\right\rangle\right|-1\right)^{2} \geq c \operatorname{dist}^{2}(D, \mathcal{S} O(2))
$$

for a diagonal matrix $D$ and $\tilde{\eta}^{k}=R \eta^{k}$. Note that if $\theta$ is the angle associated to the rotation $R$ than $\tilde{\eta}^{1}=$ $(\cos (\theta), \sin (\theta)), \tilde{\eta}^{2}=\left(\cos \left(\theta+\frac{\pi}{3}\right), \sin \left(\theta+\frac{\pi}{3}\right)\right)$ and $\tilde{\eta}^{3}=\left(\cos \left(\theta+\frac{2 \pi}{3}\right), \sin \left(\theta+\frac{2 \pi}{3}\right)\right)$.

We argue as above and introduce the function $g_{\theta}$ defined as

$$
g_{\theta}(x, y)=\sum_{k=1}^{3}\left(\sqrt{(1+x)^{2} \cos ^{2}\left(\theta_{k}\right)+(1+y)^{2} \sin ^{2}\left(\theta_{k}\right)}-1\right)^{2}
$$

with $\theta_{1}=\theta, \theta_{2}=\theta+\frac{\pi}{3}$ and $\theta_{3}=\theta+\frac{2 \pi}{3}$. We carry on the same computation and Taylor expansion as above and find

$$
\begin{aligned}
g_{\theta}(x, y) & =\sum_{k=1}^{3}\left(x \cos ^{2}\left(\theta_{k}\right)+y \sin ^{2}\left(\theta_{k}\right)\right)^{2}+o\left(x^{2}+y^{2}\right) \\
& =\sum_{k=1}^{3}\left(x^{2} \cos ^{4}\left(\theta_{k}\right)+y^{2} \sin ^{4}\left(\theta_{k}\right)+2 x y \sin ^{2}\left(\theta_{k}\right) \cos ^{2}\left(\theta_{k}\right)\right)+o\left(x^{2}+y^{2}\right) .
\end{aligned}
$$

We rely on the identities

$$
\sum_{k=1}^{3} \cos ^{4}\left(\theta_{k}\right)=\frac{9}{8}=\sum_{k=1}^{3} \sin ^{4}\left(\theta_{k}\right), \quad \sum_{k=1}^{3} \sin ^{2}\left(\theta_{k}\right) \cos ^{2}\left(\theta_{k}\right)=\frac{3}{8}
$$

and get

$$
g_{\theta}(x, y)=\frac{9}{8}\left(x^{2}+y^{2}\right)+\frac{3}{4} x y+o\left(x^{2}+y^{2}\right) .
$$

As the estimate is independent of the choice of $\theta$ (and so also of $R$ ) the conclusion follows.

Lemma 4.4. Let $\psi(A):=\operatorname{dist}^{2}(A, \mathcal{S O}(2))$ and let $A \in \mathcal{M}(2 \times 2, \mathbb{R})$ be such that $\psi^{q c}(A)=0$. Then $A \in \mathcal{S} O(2)$.

Proof. For $A \in \mathcal{M}(2 \times 2, \mathbb{R})$ fixed, taking Proposition 2.4 into account, let $\left(\varphi_{k}\right)_{k}$ be contained in $W_{0}^{1, \infty}\left(Q, \mathbb{R}^{2}\right)$ with $Q$ a unitary cube and such that

$$
\lim _{k} f_{Q} \operatorname{dist}^{2}\left(A+\nabla \varphi_{k}(x), \mathcal{S} O(2)\right) \mathrm{d} x=\psi^{q c}(A) .
$$

For almost every $x \in Q$ we select $B_{k}(x) \in \operatorname{argmin} \operatorname{dist}\left(A+\nabla \varphi_{k}(x), \mathcal{S} O(2)\right)$; that is,

$$
\left|A+\nabla \varphi_{k}(x)-B_{k}(x)\right|=\operatorname{dist}\left(A+\nabla \varphi_{k}(x), \mathcal{S} O(2)\right) .
$$


For any $k \in \mathbb{N}, x \rightarrow B_{k}(x)$ is bounded in $L^{2}(Q, \mathcal{S O}(2))$ uniformly on $k$ and we have

$$
\int_{Q}\left|\nabla \varphi_{k}\right|^{2} \mathrm{~d} x \leq 2 \int_{Q}\left|A-B_{k}(x)\right|^{2} \mathrm{~d} x+2 \int_{Q} \operatorname{dist}^{2}\left(A+\nabla \varphi_{k}(x), \mathcal{S} O(2)\right) \mathrm{d} x \leq c .
$$

Hence, it is not restrictive to assume that $\varphi_{k} \rightarrow \varphi_{\infty}$ and $B_{k} \rightarrow B_{\infty}$ weakly in $W_{0}^{1,2}\left(Q, \mathbb{R}^{2}\right)$ and $L^{2}\left(Q, \mathbb{R}^{2}\right)$, respectively. By lower semicontinuity, using that $\psi^{q c}(A)=0$, we also get

$$
0=\psi^{q c}(A)=\lim _{k} f_{Q}\left|A+\nabla \varphi_{k}(x)-B_{k}(x)\right|^{2} \mathrm{~d} x \geq f_{Q}\left|A+\nabla \varphi_{\infty}(x)-B_{\infty}(x)\right|^{2} \mathrm{~d} x .
$$

Thus, $\psi\left(A+\nabla \varphi_{\infty}(x)\right)=0$ for almost every $x \in Q$; that is, $\langle A, x\rangle+\varphi_{\infty}(x)=\langle R(x), x\rangle$ for some measurable choice of $R(x) \in \mathcal{S} O(2)$. The classical Liouville rigidity theorem, extended to $W^{1,2}\left(Q, \mathbb{R}^{2}\right)$ by Reshetnyak [22], yields in turn that $\langle A, x\rangle+\varphi_{\infty}(x)=\langle R, x\rangle$ for a fixed rotation $R$. As $\varphi \in W_{0}^{1,2}\left(Q, \mathbb{R}^{2}\right)$ and $\nabla \varphi$ is constant in $Q$, we infer by the boundary conditions that $\varphi_{\infty}=0$ and $A=R$.

We note that in the proof of Proposition 4.1 any choice of the parameter $s \in(0,1)$ ensures the validity of estimate (4.7). Moreover, assuming that $u_{\varepsilon}$ converges to a given $u$ in $L^{1}\left(\Omega ; \mathbb{R}^{2}\right)$, any sequence $v_{\varepsilon}=v_{\varepsilon}(s)$, defined in (4.3), still converges to $u$ weakly in $S B V\left(\Omega, \mathbb{R}^{2}\right)$ and the energy contribution is proportional to $\mathcal{H}^{1}\left(S_{v_{\varepsilon}}\right)$. Since $S_{v_{\varepsilon}}$ is a sequence of rectifiable sets with normal coordinates, this suggests that in the passage to the liminf on $\varepsilon$ on the surface part of the right-hand side of (4.7) we may obtain a lower bound with a surface-type energy maintaining the symmetries of the hexagonal lattice. In the sequel we build the correct surface energy arguing by pairwise interactions along lattice directions and exploiting more refined techniques in geometric measure theory.

Before entering into details we need to introduce some more tools. Let $\psi: \mathbb{R}^{2} \rightarrow[0,+\infty)$ be the 1-homogeneous map such that $\{\psi \leq 1\}$ coincides with the convex hull of the set $\mathrm{S}$ of the unitary vectors of the lattice $L_{1}$. By construction $\psi$ is a norm on $\mathbb{R}^{2}$ and we may consider its dual norm $\psi^{*}: \mathbb{R}^{2} \rightarrow[0,+\infty)$ given by the polar function of $\psi$ defined as

$$
\psi^{*}(\nu)=\sup _{|\xi|=1} \frac{\langle\nu, \xi\rangle}{\psi(\xi)}=\sup \{\langle\nu, \xi\rangle: \psi(\xi)=1\} .
$$

An easy computation shows also that

$$
\psi^{*}(\nu)=\sup _{k=1,2,3}\left|\left\langle\nu, \eta^{k}\right\rangle\right|
$$

and $\psi^{*}$ is the 1-homogeneous functions whose unitary ball is the convex hull of the coordinate directions $\mathrm{D}$ scaled by a factor $2 / \sqrt{3}$. In addition the following lemma holds true.

Lemma 4.5. If $\psi^{*}$ is as above then $2 \psi^{*}(\nu)=\sum_{k=1}^{3}\left|\left\langle\nu, \eta^{k}\right\rangle\right|$ for all $\nu \in \mathbb{R}^{2}$.

Proof. A direct computation shows that the inequality holds true as an equality for $\nu \in \mathrm{D}$. Hence one can argue locally in each sector of amplitude $\pi / 3$ by using the linearity of $\psi^{*}$ on such portions of $\mathbb{R}^{2}$ and the result for the coordinate directions.

By means of Lemma 4.5 in the next proposition we will provide a lower bound on $\Gamma$-liminf of $E_{\varepsilon}$ by an anisotropic surface energy. Since by Proposition 4.1 the $\Gamma$-lim inf of $E_{\varepsilon}$ is finite only on $S B V\left(\Omega, \mathbb{R}^{2}\right)$ we prove the estimate in that functional space.

Proposition 4.6. Let $\varphi=J_{\infty}(4 / \sqrt{3}) \psi^{*}$, then for any $u \in S B V\left(\Omega, \mathbb{R}^{2}\right)$ it holds

$$
\Gamma-\liminf _{\varepsilon \rightarrow 0^{+}} E_{\varepsilon}(u) \geq \int_{S_{u}} \varphi\left(\nu_{u}\right) \mathrm{d} \mathcal{H}^{1} .
$$


Proof. Let $\left\{u_{\varepsilon}\right\}_{\varepsilon} \subset L^{1}\left(\Omega ; \mathbb{R}^{2}\right)$ be fixed with $u_{\varepsilon} \rightarrow u$ in $L^{1}\left(\Omega, \mathbb{R}^{2}\right)$ and $\left\|u_{\varepsilon}\right\|_{L^{\infty}\left(\Omega, \mathbb{R}^{2}\right)}+E_{\varepsilon}\left(u_{\varepsilon}\right) \leq c$. It is not restrictive to assume that $\liminf _{\varepsilon \rightarrow 0^{+}} E_{\varepsilon}\left(u_{\varepsilon}\right)=\lim _{\varepsilon \rightarrow 0^{+}} E_{\varepsilon}\left(u_{\varepsilon}\right)$ and $u_{\varepsilon} \rightarrow u$ almost everywhere in $\Omega$ as well. Note that by Proposition $4.1 u$ is a piecewise rigid deformation with $\mathcal{H}^{1}\left(S_{u}\right)<+\infty$.

We will proceed by a slicing technique, performed only in the lattice directions $\eta_{1}, \eta_{2}, \eta_{3}$, in order to obtain the estimate

$$
\lim _{\varepsilon \rightarrow 0^{+}} E_{\varepsilon}\left(u_{\varepsilon}\right) \geq \sum_{k=1}^{3} \frac{2}{\sqrt{3}} \int_{S_{u}}\left|\left\langle\nu_{u}(x), \eta^{k}\right\rangle\right| \mathrm{d} \mathcal{H}^{1}(x) .
$$

To this end we observe that we can split the energies $E_{\varepsilon}$ by accounting separately for the contribution of pairs $(i, j)$ with $j-i= \pm \eta^{k} \varepsilon$ and letting $k$ vary in $\{1,2,3\}$. More precisely, for $k=1,2,3$ let

$$
L_{\varepsilon}^{k}=\left\{i \in L_{\varepsilon}:\left[i, i+\varepsilon \eta^{k}\right] \subset \Omega\right\}
$$

and define for $v \in \mathcal{A} d_{\varepsilon}(\Omega)$

$$
E_{\varepsilon}^{k}(v)=\sum_{i \in L_{\varepsilon}^{k}} \varepsilon J\left(\left|\frac{v(\mathbf{i})-v\left(\mathbf{i}+\varepsilon \eta^{k}\right)}{\varepsilon}\right|\right) ;
$$

An easy computation shows that

$$
E_{\varepsilon}\left(u_{\varepsilon}\right)=E_{\varepsilon}^{1}\left(u_{\varepsilon}\right)+E_{\varepsilon}^{2}\left(u_{\varepsilon}\right)+E_{\varepsilon}^{3}\left(u_{\varepsilon}\right)
$$

thus it is enough to prove that for any $k$

$$
\lim _{\varepsilon \rightarrow 0^{+}} E_{\varepsilon}^{1}\left(u_{\varepsilon}\right) \geq \frac{2}{\sqrt{3}} \int_{S_{u}}\left|\left\langle\nu_{u}(x), \eta^{k}\right\rangle\right| \mathrm{d} \mathcal{H}^{1}(x) .
$$

Since the lattices $L_{\varepsilon}$, and hence the energies appearing in both sides of (4.12), are invariant under rotations of $\pi / 3$ we confine our attention to prove (4.12) for $k=1$ and $\eta^{1}=e_{1}$.

Note that the functionals $E_{\varepsilon}^{1}$ consist of a superposition of 1-dimensional discrete energies related to the sublattices $\varepsilon \mathbb{Z} \times\{m \varepsilon \sqrt{3} / 2\}$ for $m$ even varying in $\mathbb{Z}$ and to the lattices $\varepsilon(\mathbb{Z}+(1 / 2)) \times\{m \varepsilon \sqrt{3} / 2\}$ for $m$ odd. Hence, as a first step we will use a usual separation of scale argument on these 1-dimensional discrete energies in order to rewrite them in a 1-dimensional integral form and then we glue the information back to the 2 -dimensional setting by a slicing procedure. More precisely, for any $m \in \mathbb{Z}$ set

$$
S_{m}=\mathbb{R} \times[m \varepsilon \sqrt{3} / 2,(m+1) \varepsilon \sqrt{3} / 2) .
$$

Note that the stripes $S_{m} \cap \Omega$ give a partition of $\Omega$. For any $s \in(0,1)$ we will construct a sequence $\left\{w_{\varepsilon}^{s}\right\} \subset$ $S B V\left(\Omega, \mathbb{R}^{2}\right)$ with 1-dimensional profile along the direction $e_{1}$ and depending only in the first variable in each stripe. To that end, let $s \in(0,1)$ be fixed and set

$$
I_{\varepsilon}=\left\{\mathrm{i} \in L_{\varepsilon}^{1}: J\left(\left|u(\mathrm{i})-u\left(\mathrm{i}+\varepsilon e_{1}\right)\right| / \varepsilon\right) \geq s J_{\infty}\right\}
$$

(for the sake of notation we drop the dependence on $s$ in what follows). By definition of $I_{\varepsilon}$ it holds

$$
E_{\varepsilon}^{1}\left(u_{\varepsilon}\right) \geq s J_{\infty} \varepsilon \#\left(I_{\varepsilon}\right) .
$$

For any $m \in \mathbb{Z}$ define $w_{\varepsilon}$ on $\mathbb{R} \times\{m \varepsilon \sqrt{3} / 2\}$ to be equal to the value $u_{\varepsilon}(\mathbf{i})$ on $\left(\mathbf{i}_{1}, \dot{i}_{1}+\varepsilon\right) \times\{m \varepsilon \sqrt{3} / 2\}$ if $i=\left(i_{1}, i_{2}\right) \in I_{\varepsilon}$ and to be the affine interpolation of the values $u_{\varepsilon}(i), u_{\varepsilon}\left(i+\varepsilon e_{1}\right)$ on any other interval $\left(\mathbf{i}_{1}, \dot{i}_{1}+\varepsilon\right) \times\{m \varepsilon \sqrt{3} / 2\}$. Eventually, extend $w_{\varepsilon}$ on $S_{m} \cap \Omega$ as $w_{\varepsilon}\left(x_{1}, x_{2}\right)=w_{\varepsilon}\left(x_{1}, m \varepsilon \sqrt{3} / 2\right)$.

We claim that $w_{\varepsilon} \rightarrow u$ in $L^{1}\left(\Omega, \mathbb{R}^{2}\right)$ and, up to subsequences, also almost everywhere in $\Omega$. Indeed, as a consequence of Lemma 2.11 in [1], the convergence of $u_{\varepsilon} \rightarrow u$ in $L^{1}\left(\Omega, \mathbb{R}^{2}\right)$ implies the convergence to $u$ in $L^{1}\left(\Omega, \mathbb{R}^{2}\right)$ also of the piecewise-constant interpolations of the values of the nodes $\left\{u_{\varepsilon}(i)\right\}$, and, in turn, also the convergence of $w_{\varepsilon} \rightarrow u$ in $L^{1}\left(\Omega, \mathbb{R}^{2}\right)$ (see also Rem. 3.2). 
Estimate (4.13) and the fact that $\nabla w_{\varepsilon}$ belongs pointwise to the compact set $\left\{\xi \in \mathbb{R}^{2}: J(\xi)<s J_{\infty}\right\}$, yield that

$$
\int_{\Omega}\left|\nabla w_{\varepsilon}\right|^{2} \mathrm{~d} x+\varepsilon \#\left(I_{\varepsilon}\right) \leq c .
$$

Taking into account that

$$
\#\left(I_{\varepsilon}\right)=\sum_{m \in \mathbb{Z}} \#\left(I_{\varepsilon} \cap S_{m}\right)
$$

and also that

$$
\#\left(I_{\varepsilon} \cap S_{m}\right)=\#\left(S\left(w_{\varepsilon}^{e_{1}, y}\right)\right)
$$

for all $y \in S_{m} \cap \Pi^{e_{1}},(4.13)$ can be rewritten as

$$
E_{\varepsilon}^{1}\left(u_{\varepsilon}\right) \geq s J_{\infty} \frac{2}{\sqrt{3}} \int_{\Pi^{e_{1}}} \#\left(S\left(w_{\varepsilon}^{e_{1}, y}\right)\right) \mathrm{d} \mathcal{H}^{1}(y) .
$$

By passing to the liminf in both sides of (4.15) and by applying Fatou's Lemma we get

$$
\lim _{\varepsilon \rightarrow 0^{+}} E_{\varepsilon}^{1}\left(u_{\varepsilon}\right) \geq s J_{\infty} \frac{2}{\sqrt{3}} \int_{\Pi^{e_{1}}} \liminf _{\varepsilon \rightarrow 0^{+}} \#\left(S\left(w_{\varepsilon}^{e_{1}, y}\right)\right) \mathrm{d} \mathcal{H}^{1}(y) .
$$

On the other hand, estimate (4.14) ensures that for $\mathcal{H}^{1}$-almost every $y \in \Pi^{e_{1}}$ the sequence $w_{\varepsilon}^{e_{1}, y}$ is precompact in $S B V\left(\Omega^{e_{1}, y}, \mathbb{R}^{2}\right)$ and converges in measure to $u^{e_{1}, y}$. Hence, by the 1-dimensional analogue of the lowersemicontinuity Theorem 2.5 we infer that

$$
\lim _{\varepsilon \rightarrow 0^{+}} E_{\varepsilon}^{1}\left(u_{\varepsilon}\right) \geq s J_{\infty} \frac{2}{\sqrt{3}} \int_{\Pi^{e_{1}}} \#\left(S\left(u^{e_{1}, y}\right)\right) \mathrm{d} \mathcal{H}^{1}(y)
$$

Moreover, thanks to Theorem 2.6(a) with $g=1$, we have

$$
\int_{\Pi^{e_{1}}} \#\left(S\left(u^{e_{1}, y}\right)\right) \mathrm{d} \mathcal{H}^{1}(y)=\int_{S_{u}}\left|\left\langle\nu_{u}(x), e_{1}\right\rangle\right| \mathrm{d} \mathcal{H}^{1}(x) .
$$

Letting $s \rightarrow 1$ concludes the proof of claim (4.11). Eventually, Lemma 4.5 yields (4.10).

\section{UpPer estimates FOR A CLASS OF "OPENING CRACKS"}

In this section we show the optimality of the bound (4.10) provided in Proposition 4.6 for a class of functions with 'opening cracks' with respect to the anisotropies inherited by the lattice.

In order to clarify the parameters playing a role in the asymptotic behaviour of $E_{\varepsilon}$, we prefer to deal with the case where only two rotations $R^{+}, R^{-}$are involved in the target deformation $u$ first. In this setting the crack $S_{u}$ splits $\Omega$ into two regions $\Omega^{+}, \Omega^{-}$, in general not connected, each one underlying a rigid motion. We will show that in this case the $\Gamma$-limsup of $E_{\varepsilon}$ is finite even if the request of orientation-preserving recovery sequences affects substantially the form of the limit energy.

In this process a relevant condition that translates the positive-determinant constraint through the crack is the following:

$$
\left\langle u^{+}(x)-u^{-}(x), R^{ \pm} \nu\right\rangle \geq 0 .
$$

In fact, the inequality above ensures that any small triangle crossing the fracture site maintains positive (non negative) area in the codomain. Actually, due to the discrete environment, condition (5.1) has to be assumed for $\nu \in \mathrm{D}$, since the only triangles entering in the construction have sides parallel to the directions lying in $\mathrm{S}$.

We start by proving the following characterization of the $\Gamma$-limsup whenever $S_{u}$ is contained in a straight line and (5.1) holds. 
Proposition 5.1. For $R^{ \pm} \in \mathcal{S O}(2)$ and $\bar{x}, \nu, q^{ \pm} \in \mathbb{R}^{2}$ with $\nu \in \mathrm{D}$, let $u$ be defined as

$$
u(x)=\left\{\begin{array}{lll}
R^{+} x+q^{+} & \text {if } & \langle x-\bar{x}, \nu\rangle>0, x \in \Omega \\
R^{-} x+q^{-} & \text {if } & \langle x-\bar{x}, \nu\rangle \leq 0, x \in \Omega,
\end{array}\right.
$$

so that $\nu_{u}=\nu \mathcal{H}^{1}$-almost everywhere. Assume that $R^{+} \neq-R^{-}$and for $\mathcal{H}^{1}$-almost every $x \in S_{u} R^{ \pm}, q^{ \pm}, \nu$ satisfy

$$
\left\langle u^{+}(x)-u^{-}(x), R^{ \pm} \nu\right\rangle \geq 0,
$$

where $u^{+}(x)=R^{+} x+q^{+}, u^{-}(x)=R^{-} x+q^{-}$, then

$$
\Gamma-\limsup _{\varepsilon \rightarrow 0^{+}} E_{\varepsilon}(u) \leq \int_{S_{u}} \varphi(\nu) \mathrm{d} \mathcal{H}^{1}=2 J_{\infty} \mathcal{H}^{1}\left(S_{u}\right) .
$$

Proof. We claim that, thanks to the hypothesis $R^{+} \neq-R^{-}$, we may assume the stronger separation hypothesis

$$
\left\langle u^{+}(x)-u^{-}(x), R^{ \pm} \nu\right\rangle \geq \delta>0
$$

for some fixed $\delta>0$ and for $\mathcal{H}^{1}$-almost every $x \in S_{u}$. Indeed, if (5.5) does not hold, it is enough to choose a vector $v$ such that $\left\langle v, R^{ \pm} \nu\right\rangle>0$ (set for instance $v=R^{+} \nu+R^{-} \nu$ ) and consider the sequence $u_{\delta}$ defined replacing $q^{+}$with $q^{+}+\delta v$ in (5.2). Then $S_{u_{\delta}}=S_{u}, u_{\delta} \rightarrow u$ in $L^{2}\left(\Omega, \mathbb{R}^{2}\right)$ and the left-hand side term of (5.4) is continuous along such a sequence, while the right-hand side is independent of $\delta$.

Condition (5.5) plays a key role in showing that the sequence $\left(u_{\varepsilon}\right)$ defined as the pointwise interpolation of $u$ in the nodes of the lattice $L_{\varepsilon} \cap \Omega$ is admissible and accounts for the desired $\Gamma$-limsup estimate. We then simply define $u_{\varepsilon}(\mathbf{i})=u(\mathbf{i})$ for any $i \in L_{\varepsilon} \cap \Omega$. Since $u_{\varepsilon} \rightarrow u$ in $L^{1}\left(\Omega, \mathbb{R}^{2}\right)$, it remains to check that the positive-determinant constraint is satisfied in any triangle contained in $\Omega$.

Before proceeding with the computation we label the triangles 'crossing the fracture site'. By rotational and translational invariance we may assume $\nu=(0,1)$ so that $S_{u}=\left\{x=\left(x_{1}, x_{2}\right) \in \Omega: x_{2}=\bar{x}_{2}\right\}$. Let $N_{\varepsilon}:=\left[2 \bar{x}_{2} / \sqrt{3} \varepsilon\right]$ and set

$$
\mathcal{D}_{\varepsilon}=\left\{m \in \mathbb{Z}:\left(m \varepsilon, \sqrt{3} N_{\varepsilon} \varepsilon / 2\right) \in L_{\varepsilon} \cap \Omega\right\}
$$

and

$$
\dot{i}_{m}=\left(m \varepsilon, \sqrt{3} N_{\varepsilon} \varepsilon / 2\right) \quad \text { for } m \in \mathcal{D}_{\varepsilon} .
$$

Note that $u$ coincides with a 'positive' rotation on the vertices of those triangles not contained in the strip

$$
\mathcal{S}=\left\{x: \sqrt{3} N_{\varepsilon} \varepsilon / 2 \leq x_{2} \leq \sqrt{3}\left(N_{\varepsilon}+1\right) \varepsilon / 2\right\} .
$$

Hence, in order to ensure that $u_{\varepsilon} \in \mathcal{A} d_{\varepsilon}(\Omega)$, it suffices to check condition (5.3) for triangles $\mathrm{T}$ with vertices respectively $\dot{i}_{m}, \dot{i}_{m}+\varepsilon \eta^{1}, \dot{i}_{m}+\varepsilon \eta^{2}$ and $\dot{i}_{m}, \dot{i}_{m}+\varepsilon \eta^{2}, \dot{i}_{m}+\varepsilon\left(\eta^{2}-\eta^{1}\right)$ (see Fig. 3 ). This leads to proving the following inequalities:

$$
\begin{gathered}
\left\langle\left(u^{-}\left(\mathbf{i}_{m}+\varepsilon \eta^{1}\right)-u^{-}\left(\dot{i}_{m}\right)\right)^{\perp}, u^{+}\left(\dot{i}_{m}+\varepsilon \eta^{2}\right)-u^{-}\left(\mathbf{i}_{m}\right)\right\rangle \geq 0, \\
\left\langle\left(u^{+}\left(\dot{i}_{m}+\varepsilon \eta^{2}\right)-u^{+}\left(\dot{i}_{m}+\varepsilon\left(\eta^{2}-\eta^{1}\right)\right)^{\perp}, u^{+}\left(\dot{i}_{m}+\varepsilon \eta^{2}\right)-u^{-}\left(\dot{i}_{m}\right)\right\rangle \geq 0\right.
\end{gathered}
$$

for $m \in \mathcal{D}_{\varepsilon}$. These can be compactly rewritten as

$$
\varepsilon\left\langle R^{ \pm} \nu, u^{+}\left(\dot{i}_{m}+\varepsilon \eta^{2}\right)-u^{-}\left(\dot{i}_{m}\right)\right\rangle \geq 0 .
$$

After recalling that we are choosing the counter-clockwise orthogonal vector, that $u^{ \pm}$are affine and that $\hat{x} \in S_{u}$, we claim that these conditions are fulfilled for $\varepsilon$ small enough. Indeed, for any infinite collection of indices $\left\{m_{\varepsilon}\right\} \subset \mathbb{Z}$ with $m_{\varepsilon} \in \mathcal{D}_{\varepsilon}$, up to subsequences, we may assume that $i_{m_{\varepsilon}} \rightarrow \hat{x}$ as $\varepsilon \rightarrow 0+$. As a consequence 

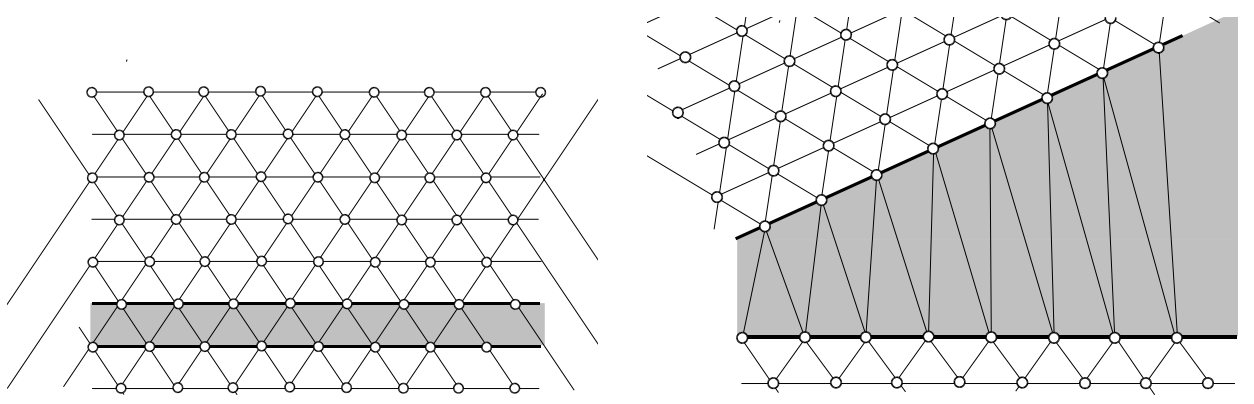

Figure 3. Triangles deformed by the pointwise interpolation.

$u^{ \pm}\left(\mathbf{i}_{m_{\varepsilon}}+\varepsilon \eta^{1}\right) \rightarrow u^{ \pm}(\hat{x})$ and $u^{ \pm}\left(\mathbf{i}_{m_{\varepsilon}}\right) \rightarrow u^{ \pm}(\hat{x})$, with $\hat{x}_{2}=\bar{x}_{2}$. Thus, assuming that (5.6) are violated for such a sequence of indices $m_{\varepsilon}$ will lead to a contradiction to (5.5) when passing to the limit as $\varepsilon \rightarrow 0+$.

Eventually, we compute the asymptotic value of $E_{\varepsilon}\left(u_{\varepsilon}\right)$ to show that $\left(u_{\varepsilon}\right)$ is a recovery sequence for the surface energy $\int_{S_{u}} \varphi\left(\nu_{u}\right) \mathrm{d} \mathcal{H}^{1}$. The energy contribution in $E_{\varepsilon}\left(u_{\varepsilon}\right)$ reduces to those pairs of indices one of which of type $i_{m}$ with $m \in \mathcal{D}_{\varepsilon}$; i.e.,

$$
\begin{aligned}
E_{\varepsilon}\left(u_{\varepsilon}\right)= & \sum_{m \in \mathcal{D}_{\varepsilon}} \varepsilon J\left(\frac{\left|u^{+}\left(\mathbf{i}_{m}+\varepsilon \eta^{2}\right)-u^{-}\left(\mathbf{i}_{m}\right)\right|}{\varepsilon}\right) \\
& +\sum_{m \in \mathcal{D}_{\varepsilon}} \varepsilon J\left(\frac{\left|u^{+}\left(\mathbf{i}_{m}+\varepsilon\left(\eta^{2}-\eta^{1}\right)\right)-u^{-}\left(\mathbf{i}_{m}\right)\right|}{\varepsilon}\right) .
\end{aligned}
$$

If $R^{+}=R^{-}$and $q^{+}=q^{-}$then

$$
E_{\varepsilon}\left(u_{\varepsilon}\right)=\sum_{m \in \mathcal{D}_{\varepsilon}} \varepsilon J\left(\left|R^{+} \eta^{2}\right|\right)+\sum_{m \in \mathcal{D}_{\varepsilon}} \varepsilon J\left(\left|R^{+}\left(\eta^{2}-\eta^{1}\right)\right|\right)=0
$$

and the thesis is proved as $S_{u}=\emptyset$. If $R^{+} \neq R^{-}$and $q^{+} \neq q^{-}$then

$$
\begin{aligned}
E_{\varepsilon}\left(u_{\varepsilon}\right)= & \sum_{m \in \mathcal{D}_{\varepsilon}} \varepsilon J\left(\left|\frac{\left(R^{+}-R^{-}\right)\left(\mathbf{i}_{m}\right)}{\varepsilon}+\frac{q^{+}-q^{-}}{\varepsilon}+\left(R^{+}-R^{-}\right) \eta^{2}\right|\right) \\
& +\sum_{m \in \mathcal{D}_{\varepsilon}} \varepsilon J\left(\left|\frac{\left(R^{+}-R^{-}\right)\left(\mathbf{i}_{m}\right)}{\varepsilon}+\frac{q^{+}-q^{-}}{\varepsilon}+\left(R^{+}-R^{-}\right)\left(\eta^{2}-\eta^{1}\right)\right|\right)
\end{aligned}
$$

and, taking into account that $i_{m} / \varepsilon=\left(m, \sqrt{3} N_{\varepsilon} / 2\right)$, the argument of $J$ in all the terms in the summation above tends to $+\infty$. Hence,

$$
E_{\varepsilon}\left(u_{\varepsilon}\right)=2 \varepsilon \# \mathcal{D}_{\varepsilon}\left(J_{\infty}+o(1)\right)
$$

Passing to the limit as $\varepsilon \rightarrow 0$ and plugging the equality

$$
\lim _{\varepsilon \rightarrow 0^{+}} \varepsilon \# \mathcal{D}_{\varepsilon}=\mathcal{H}^{1}\left(S_{u}\right)
$$

in (5.7) the conclusion is achieved.

The next proposition generalizes the result of Proposition 5.1 in the case when only two rotations are involved in the deformation and $S_{u}$ consists of finite line segments having normals in D and the stronger open crack condition (5.5) is satisfied along the jump set $S_{u}$. 
Proposition 5.2. Let $R^{ \pm} \in \mathcal{S} O(2)$ and $q^{ \pm} \in \mathbb{R}^{2}$ be given and let

$$
u(x)=\left(R^{+} x+q^{+}\right) \chi_{\Omega^{+}}(x)+\left(R^{-} x+q^{-}\right) \chi_{\Omega^{-}}(x)
$$

where $\Omega^{+}, \Omega^{-}$is a partition of $\Omega$ with $\partial^{*} \Omega^{+}=\partial^{*} \Omega^{-}=S$, with $S \subset \Omega$ a finite connected union of line segments with normal belonging to $\mathrm{D}$. Assume that for $\mathcal{H}^{1}$-almost every $x \in S$

$$
\left\langle u^{+}(x)-u^{-}(x), R^{ \pm} \nu(x)\right\rangle \geq \delta
$$

for some $\delta>0[$; then

$$
\Gamma-\limsup _{\varepsilon \rightarrow 0^{+}} E_{\varepsilon}(u) \leq \int_{S} \varphi(\nu(x)) \mathrm{d} \mathcal{H}^{1}=2 J_{\infty} \mathcal{H}^{1}(S) .
$$

Proof. Let $S=\bigcup_{k \in \mathcal{I}} S_{k}$ with $S_{k}=\left[z^{k}, w^{k}\right]$ for $z^{k}, w^{k} \in \mathbb{R}^{2}$, having constant normal $\nu_{k}$ lying in D and satisfying $w^{k}=z^{k+1}$ for any $k$. Thanks to the strong opening hypothesis (5.9), we may replace $S$ with $S^{\varepsilon}=\cup_{k \in \mathcal{I}} S_{k}^{\varepsilon}$ with $S_{k}^{\varepsilon}=\left[i_{\varepsilon}^{k}, j_{\varepsilon}^{k}\right]$ for $i_{\varepsilon}^{k}, j_{\varepsilon}^{k} \in L_{\varepsilon} \cap \Omega$, having constant normal $\nu_{k}$ lying in D and satisfying $\mathrm{j}_{\varepsilon}^{k}=\mathrm{i}_{\varepsilon}^{k+1}$ for any $k$. More in detail, $S_{k}^{\varepsilon}$ can be selected inductively by applying a finite number of small translations to the line segments $S_{k}$ of $S$, together with replacing their endpoints with points on the lattice $L_{\varepsilon}$ so that the normal to $S_{k}^{\varepsilon}$ coincides with the normal to $S_{k}$. Note that, thanks to the boundary regularity of $\omega$ for any $k \in \mathcal{I}$ we have $\left|\mathcal{H}^{1}\left(S_{k}^{\varepsilon}\right)-\mathcal{H}^{1}\left(S_{k}\right)\right| \leq \varepsilon$. We claim that a recovery sequence is provided defining $u_{\varepsilon}(\mathrm{i})=v_{\varepsilon}^{+}(\mathrm{i})$ for $\mathrm{i} \in L_{\varepsilon} \cap \Omega$, where $v_{\varepsilon}$ is defined as in (5.8) replacing $\Omega^{+}, \Omega^{-}$with $\Omega_{\varepsilon}^{+}, \Omega_{\varepsilon}^{-}$such that $\partial^{*} \Omega_{\varepsilon}^{+}=\partial^{*} \Omega_{\varepsilon}^{-}=S^{\varepsilon}$. Indeed, for any triangle $T \in \mathcal{T}_{\varepsilon}^{c}$ intersecting $S^{\varepsilon}$ there exists a unique $k \in \mathcal{I}$ such that either $T \cap S^{\varepsilon}$ is a single point in $S_{k}^{\varepsilon}$ or $T \cap S^{\varepsilon}=\left[i, i \pm \varepsilon \nu_{k}^{\perp}\right]$ for some $i \in L_{\varepsilon} \cap \Omega$. In both cases one may perform the same computation as in Proposition 5.1, and deduce that $u_{\varepsilon}$ satisfies the positive-determinant constraint. Eventually a direct computation shows that $E_{\varepsilon}\left(u_{\varepsilon}\right)=\left(J_{\infty}+o(1)\right) \sum_{k \in \mathcal{I}} \varphi\left(\nu_{k}\right) \mathcal{H}^{1}\left(S_{k}^{\varepsilon}\right)$ and this tends to $J_{\infty} \sum_{k \in \mathcal{I}} \varphi\left(\nu_{k}\right) \mathcal{H}^{1}\left(S_{k}\right)$ as $\varepsilon \rightarrow 0$.

The previous approach can be pushed further to obtain the optimality of the bound (4.10) also for $S_{u}$ consisting of a line with normal $\nu \notin \mathrm{D}$. In this case we need to impose the opening crack condition (5.3) in a stronger sense; i.e., (5.3) must be satisfied also along the two directions $\nu_{1}, \nu_{2} \in \mathrm{D}$ generating $\nu$ in one of the simplexes of the Wulff shape $\{\varphi \leq 1\}$.

Proposition 5.3. For $R^{ \pm} \in \mathcal{S} O(2)$ and $\bar{x}, \nu, q^{ \pm} \in \mathbb{R}^{2}$, let $u$ be defined as

$$
u(x)=\left\{\begin{array}{lcc}
R^{+} x+q^{+} & \text {if } & \langle x-\bar{x}, \nu\rangle>0, x \in \Omega \\
R^{-} x+q^{-} & \text {if } & \langle x-\bar{x}, \nu\rangle \leq 0, x \in \Omega .
\end{array}\right.
$$

Let $\nu_{1}, \nu_{2} \in \mathrm{D}$ be such that $\nu / \varphi(\nu)=\lambda \nu_{1}+(1-\lambda) \nu_{2}$ for $\lambda \in(0,1)$. Assume that $S_{u}$ is a line segment and that for $\mathcal{H}^{1}$-almost every $x \in S_{u} R^{ \pm}, q^{ \pm}, \nu$ satisfy the conditions

$$
\left\langle u^{+}(x)-u^{-}(x), R^{ \pm} \nu_{i}\right\rangle \geq \delta
$$

for $i=1,2$ and for some $\delta>0$. Then

$$
\Gamma-\limsup _{\varepsilon \rightarrow 0^{+}} E_{\varepsilon}(u) \leq \int_{S_{u}} \varphi(\nu) \mathrm{d} \mathcal{H}^{1} .
$$

Proof. The claim will be proved by an approximation argument, exploiting Proposition 5.2 and the lower semicontinuity of the $\Gamma$-limsup. Note that hypothesis (5.12) ensures that $R^{+} \neq-R^{-}$.

For all positive integers $h$ let $S_{h}$ be a finite connected union of line segments with normal equal to $\nu_{1}$ or to $\nu_{2}$ such that

$$
\lim _{h \rightarrow+\infty} \int_{S_{h}} \varphi\left(\nu_{h}(x)\right) \mathrm{d} \mathcal{H}^{1}=\int_{S_{u}} \varphi(\nu) \mathrm{d} \mathcal{H}^{1}
$$


and such the Hausdorff distance betwenn $S_{h}$ and $S_{u}$ tends to 0 . The existence of such polygonals follows from the equality $\nu^{\perp}=\lambda \varphi(\nu) \nu_{1}^{\perp}+(1-\lambda) / \varphi(\nu) \nu_{2}^{\perp}$, which implies that any vector orthogonal to $\nu$ of length $L$ is the sum of vectors orthogonal to $\nu_{1}$ and $\nu_{2}$ of length $\lambda \varphi(\nu) L$ and $(1-\lambda) \varphi(\nu) L$, respectively. Hence, for any $h \in \mathbb{N}$ fixed we subdivide the line segment $S_{u}$ into line segments of lenghts $\mathcal{H}^{1}\left(S_{u}\right) / h$ and we perform the construction above with $L=\mathcal{H}^{1}\left(S_{u}\right) / h$. Since $\varphi\left(\nu_{1}\right)=\varphi\left(\nu_{2}\right)=1$, then summing the integral of $\varphi\left(\nu_{1}\right)$ on the first segment and that of $\varphi\left(\nu_{2}\right)$ on the second one gives $\varphi(\nu) L$. Note that by the geometric construction above we infer that $d_{\mathcal{H}}\left(S_{h}, S_{u}\right) \leq c / h$.

We may also assume that each $S_{h}$ has endpoints on $\partial \Omega$ and splits $\Omega$ in two components $\Omega_{h}^{+}, \Omega_{h}^{-}$such that

$$
\lim _{h \rightarrow+\infty}\left|\Omega_{h}^{+} \triangle\{x \in \Omega:\langle x-\bar{x}, \nu\rangle>0\}\right|=0 .
$$

For $h \in \mathbb{N}$ let $u_{h}$ be the piecewise-rigid function defined as

$$
u_{h}(x)=\left(R^{+} x+q^{+}\right) \chi_{\Omega_{h}^{+}}+\left(R^{-} x+q^{-}\right) \chi_{\Omega_{h}^{-}} .
$$

Then $u_{h} \rightarrow u$ in $L^{1}\left(\Omega ; \mathbb{R}^{2}\right)$ and, by continuity, $u_{h}$ satisfies hypothesis (5.12) with a smaller positive $\delta$ and $S_{u}$ replaced by $S_{u_{h}}=S_{h}$. Applying Proposition 5.2 to each $u_{h}$ we get

$$
\Gamma-\limsup _{\varepsilon \rightarrow 0^{+}} E_{\varepsilon}\left(u_{h}\right) \leq \int_{S_{u_{h}}} \varphi\left(\nu_{h}\right) \mathrm{d} \mathcal{H}^{1} .
$$

The conclusion follows taking property (5.14) into account.

In the following we consider the case in which the jump set $S_{u}$ is a triple point with coordinate normals.

Proposition 5.4. Let $x_{0} \in \Omega$ be fixed and set

$$
\begin{aligned}
& \Omega_{1}:=\left\{x \in \Omega:\left\langle x-x_{0}, \eta^{1^{\perp}}\right\rangle \geq 0,\left\langle x-x_{0}, \eta^{2^{\perp}}\right\rangle \geq 0\right\}, \\
& \Omega_{2}:=\left\{x \in \Omega:\left\langle x-x_{0}, \eta^{2^{\perp}}\right\rangle \leq 0,\left\langle x-x_{0}, \eta^{3^{\perp}}\right\rangle \leq 0\right\}, \\
& \Omega_{3}:=\left\{x \in \Omega:\left\langle x-x_{0}, \eta^{1^{\perp}}\right\rangle \leq 0,\left\langle x-x_{0}, \eta^{3^{\perp}}\right\rangle \geq 0\right\} .
\end{aligned}
$$

Let $u$ be defined as $u(x)=\sum_{k=1}^{3} u^{k}(x) \chi_{\Omega_{k}}(x)$ with $u^{k}(x)=R^{k} x+q^{k}$ for suitable $R^{1}, R^{2}, R^{3} \in \mathcal{S} O(2)$ and $q^{1}, q^{2}, q^{3} \in \mathbb{R}^{2}$. Assume that $u$ satisfies the 'opening crack' conditions along $S_{u}=\bigcup_{k}\left(\partial \Omega_{k} \cap \Omega\right)$ :

$$
\begin{array}{lll}
\left\langle u^{1}(x)-u^{3}(x), R^{1} \eta^{1}\right\rangle \geq \delta,\left\langle u^{1}(x)-u^{3}(x), R^{3} \eta^{1^{\perp}}\right\rangle \geq \delta, & \text { on } & \partial \Omega_{1} \cap \partial \Omega_{3} \\
\left\langle u^{1}(x)-u^{2}(x), R^{2} \eta^{2^{\perp}}\right\rangle \geq \delta,\left\langle u^{1}(x)-u^{2}(x), R^{1} \eta^{2^{\perp}}\right\rangle \geq \delta, & \text { on } & \partial \Omega_{1} \cap \partial \Omega_{2} \\
\left\langle u^{3}(x)-u^{2}(x), R^{3} \eta^{3^{\perp}}\right\rangle \geq \delta,\left\langle u^{3}(x)-u^{2}(x), R^{2} \eta^{3^{\perp}}\right\rangle \geq \delta, & \text { on } & \partial \Omega_{3} \cap \partial \Omega_{2}
\end{array}
$$

for some $\delta>0$, and that $u$ satisfies the further compatibility condition

$$
\left\langle u^{1}\left(x_{0}\right)-u^{3}\left(x_{0}\right),\left(u^{2}\left(x_{0}\right)-u^{3}\left(x_{0}\right)\right)^{\perp}\right\rangle>0
$$

on the triple point $x_{0}$. Then

$$
\Gamma-\limsup _{\varepsilon \rightarrow 0^{+}} E_{\varepsilon}(u) \leq \int_{S_{u}} \varphi(\nu) \mathrm{d} \mathcal{H}^{1} .
$$

Note that if the uniform strict-positivity conditions in (5.15) is relaxed to strict positivity the perturbation argument in the proof of Proposition 5.1 may fail on points of $S_{u}$ close $\partial \Omega$. The claim of Proposition 5.4 could be then restated as holding for $u$ for which such an approximation argument works. 
Proof. Up to composing $u$ with infinitesimal translations and rotations we may assume that, for any $\varepsilon>0$ small enough, the points $x_{0}-\frac{\varepsilon}{3}\left(\eta^{1}+\eta^{2}\right), x_{0}+\frac{\varepsilon}{3}\left(\eta^{3}+\eta^{1}\right), x_{0}+\frac{\varepsilon}{3}\left(\eta^{2}-\eta^{3}\right)$ lie on the lattice $L_{\varepsilon} \cap \Omega$ and that $L_{\varepsilon} \cap S_{u}=\emptyset$. Set $u_{\varepsilon}(\mathrm{i})=u(\mathrm{i})$ for any $\mathrm{i} \in L_{\varepsilon} \cap \Omega$. Clearly $u_{\varepsilon} \rightarrow u$ in $L^{1}\left(\Omega, \mathbb{R}^{2}\right)$; it remains to check that the positive-determinant constraint is satisfied in any triangle intersecting $S_{u}$. Thanks to hypothesis (5.16) we have that there exists $\delta>0$ such that all the scalar products in (5.15) are greater than $\delta$. By performing the same computation as in the proof of Proposition 5.1 we may deduce the positive-determinant constraint to be satisfied on any triangle intersecting $S_{u}$ and not containing $x_{0}$. The only triangle left aside is then the one having vertices $x_{0}+\frac{\varepsilon \sqrt{3}}{2}\left(\eta^{2}-\eta^{1}\right), x_{0}+\frac{\varepsilon \sqrt{3}}{2}\left(\eta^{3}-\eta^{1}\right), x_{0}+\frac{\varepsilon \sqrt{3}}{2}\left(\eta^{2}+\eta^{3}\right)$. A direct computation shows that

$$
\begin{aligned}
&\left\langleu ^ { 1 } \left( x_{0}\right.\right.\left.+\frac{\varepsilon}{3}\left(\eta^{2}-\eta^{3}\right)\right)-u^{3}\left(x_{0}-\frac{\varepsilon}{3}\left(\eta^{1}+\eta^{2}\right)\right), \\
&\left.\left(u^{2}\left(x_{0}+\frac{\varepsilon}{3}\left(\eta^{1}+\eta^{3}\right)\right)-u^{3}\left(x_{0}-\frac{\varepsilon}{3}\left(\eta^{1}+\eta^{2}\right)\right)\right)^{\perp}\right\rangle \\
& \quad=\left\langle u^{1}\left(x_{0}\right)-u^{3}\left(x_{0}\right),\left(u^{2}\left(x_{0}\right)-u^{3}\left(x_{0}\right)\right)^{\perp}\right\rangle+o(1) .
\end{aligned}
$$

Hence the conclusion follows by hypothesis (5.16).

As a conclusion of this section we note that we have proved that the $\Gamma$-limit of $E_{\varepsilon}$ is described by the anisotropic fracture energy

$$
\mathcal{F}(u)=\int_{S_{u}} \varphi(\nu) \mathrm{d} \mathcal{H}^{1}
$$

on all $u$ which are piecewise rigid deformations such that $S_{u}$ consists of a finite number of lines meeting at triple points and the opening-crack conditions in Proposition 5.4 are satisfied. This description extends by continuity to all piecewise-rigid deformations $u$ that can be approximated in energy by sequences of piecewiserigid deformations $u_{h}$ satisfying such conditions.

We underline that when more complex geometries are taken into account (see Sect. 3 in [9]) the occurrence of different phenomena is highlighted. Actually, the representation of the limit energy seems to take into account several factors, not all of local nature.

\section{NeCESSARY CONDITIONS FOR OPENING CRACKS}

In Section 4 we have shown a lower bound with an anisotropic Griffith fracture energy, which is optimal on a family of displacements with an opening-crack condition on the fracture site (Sect. 5). We now show conversely that if the limit energy at a point $x_{0} \in S_{u}$ is not greater than the lower bound then necessarily the function $u$ satisfies a opening-crack condition.

We now consider discontinuity points where the fracture energy density is minimal; i.e., the inequality in (4.10) is sharp, and derive necessary conditions on the crack opening. To this end we introduce the measures

$$
\mu_{\varepsilon}=\sum_{(i, j) \in \mathcal{N}_{\varepsilon}(\Omega)} \varepsilon J\left(\left|\frac{u_{\varepsilon}(i)-u_{\varepsilon}(j)}{\varepsilon}\right|\right) \delta_{\frac{i+j}{2}} .
$$

These measures are a way to measure locally the energy $F_{\varepsilon}\left(u_{\varepsilon}\right)$. If $\mu_{\varepsilon}$ is a bounded sequence of measures and $\sup _{\varepsilon}\left\|u_{\varepsilon}\right\|_{L^{\infty}\left(\Omega, \mathbb{R}^{2}\right)}<+\infty$, then, arguing as in the proof of Proposition 4.1, we infer that $u_{\varepsilon}$ is precompact in $\operatorname{SBV}\left(\Omega, \mathbb{R}^{2}\right)$ and that $u_{\varepsilon} \rightarrow u$ in $L^{1}$. We also suppose that the weak* limit $\mu$ of $\mu_{\varepsilon}$ exists.

Proposition 6.1 (Necessity of an opening-crack condition). Let $u_{\varepsilon} \rightarrow u$, and let $x_{0} \in S_{u}$ be such that

$$
\frac{\mathrm{d} \mu}{\mathrm{d} \mathcal{H}^{1}\left\llcorner S_{u}\right.}\left(x_{0}\right) \leq \varphi\left(\nu_{u}\left(x_{0}\right)\right) .
$$




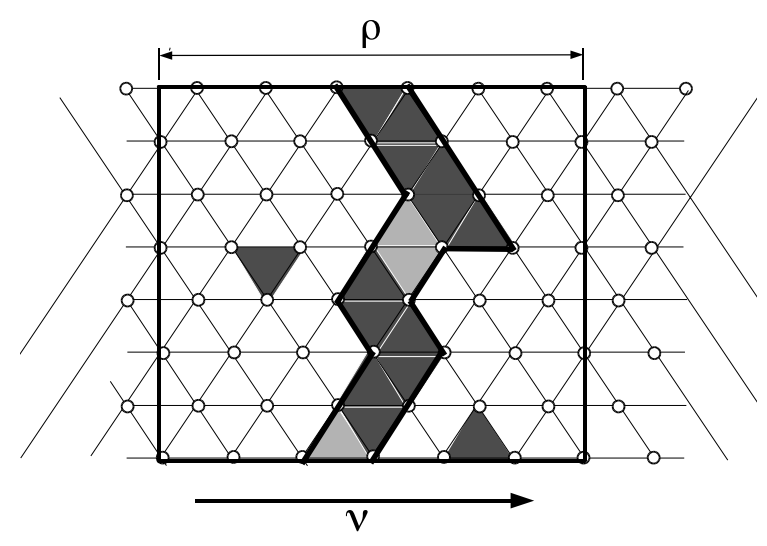

FiguRE 4. An illustration of the arguments of the proof.

Then we have

$$
\left\langle u^{+}\left(x_{0}\right)-u^{-}\left(x_{0}\right), R^{ \pm} \nu_{u}\left(x_{0}\right)\right\rangle \geq 0,
$$

where $R^{ \pm} \in \mathcal{S O}(2)$ are the two constant matrices coinciding with $\nabla u$ on both sides of $S_{u}$ at $x_{0}$.

Note that, thanks to the lower estimates in the previous section, (6.2) can be equivalently stated as an equality.

Proof. Since the proof is a little involved, we first give a short outline.

- We use a blow-up argument, examining the behaviour of energies on small squares of side-length $\rho$ centered at a point $x_{0}$ and one side oriented as $\nu_{u}\left(x_{0}\right)$. For simplicity of illustration in Figure 4 we picture such a square and the related triangulation at a fixed $\varepsilon$ when $\nu_{u}\left(x_{0}\right)=e_{1}$;

- taking $\rho$ and $\varepsilon$ small enough the values on opposite side are close to $u^{+}\left(x_{0}\right)$ and $u^{-}\left(x_{0}\right)$. This implies that there exists a path of triangles which are deformed in triangles with (two or more) large sides and that joins the two opposite sides parallel to $e_{1}$, up to adding a small percentage of triangles. Otherwise a use of the Poincarè inequality on paths joining the two sides parallel to $e_{2}$ would give a contradiction. The highly deformed triangles are pictured in dark grey in Figure 4, the additional triangles in light gray. We note that by (6.2) there is only a small percentage of highly deformed triangles that do not belong to the path described above;

- we now reason at $\rho$ small but fixed. We note that the functions defined on a unit cube by $w_{\varepsilon}^{\rho}(y)=$ $\frac{1}{\rho} u_{\varepsilon}\left(x_{0}+\rho y\right)$ except on highly-deformed triangles, where they are set to 0 , have gradients, determinants, lengths of discontinuity sets and BV norms of the tangential derivatives that satisfy uniform bounds. By the results in [3] this implies that their tangential traces weakly converge to those of $w^{\rho}(y)=\frac{1}{\rho} u\left(x_{0}+\rho y\right)$;

- using this tangential convergence and, finally, the positive-determinant constraint we describe the behaviour on the boundary of the path of highly-deformed triangles. This set is pictured in Figure 4 by two solid lines, denoted by $P_{ \pm}^{\varepsilon}$ in the proof, which converge to the jump set of $\frac{1}{\rho} u\left(x_{0}+\rho y\right)$, up to sets of very small perimeter negligible as $\rho \rightarrow 0$. Using again a Poincaré-inequality argument, we note that $w_{\varepsilon}^{\rho}(y)$ is very close to $\frac{1}{\rho} u^{ \pm}\left(x_{0}\right)$ on $P_{ \pm}^{\varepsilon}$, respectively. Integrating the positive-determinant constraint on $P_{ \pm}^{\varepsilon}$ and passing to the limit in $\varepsilon$ and then in $\rho$ we then obtain (6.3).

For the sake of brevity we will denote $\nu_{0}=\nu_{u}\left(x_{0}\right)$. Let $Q^{\nu_{0}}$ be a square centered in 0 , with side length 1 and an edge orthogonal to $\nu_{0}$. With fixed $\rho>0$ and $y \in Q^{\nu_{0}}$, let

$$
v_{\varepsilon, \rho}(y)=u_{\varepsilon}\left(x_{0}+\rho y\right),
$$


which, by definition of $S_{u}$ converges as $\varepsilon \rightarrow 0$ and subsequently $\rho \rightarrow 0$ (unless otherwise specified we will refer to convergence in this order of quantities depending on $\varepsilon$ and $\rho$ ) to the function

$$
\widetilde{u}(y)=\left\{\begin{array}{lll}
u^{+}\left(x_{0}\right) & \text { if } & \left\langle y, \nu_{0}\right\rangle \geq 0 \\
u^{-}\left(x_{0}\right) & \text { if } & \left\langle y, \nu_{0}\right\rangle<0 .
\end{array}\right.
$$

By the weak* convergence of $\mu_{\varepsilon}$ and (6.2) we deduce that

$$
E_{\varepsilon}\left(u_{\varepsilon}, Q_{\rho}^{\nu_{0}}\left(x_{0}\right)\right) \leq \rho\left(\varphi(\nu)+o_{\rho}(1)\right)+o_{\varepsilon}(1)
$$

where $Q_{\rho}^{\nu_{0}}\left(x_{0}\right)=x_{0}+\rho Q^{\nu_{0}}$, and $o_{\rho}(1)$ and $o_{\varepsilon}(1)$ are infinitesimal as $\rho \rightarrow 0$ and $\varepsilon \rightarrow 0$, respectively.

We fix $s>0$ and set

$$
\mathcal{S}_{\varepsilon}^{s}=\left\{T \in \mathcal{T}_{\varepsilon}^{c}(\Omega):\left|\frac{u_{\varepsilon}(\mathrm{i})-u_{\varepsilon}(\mathrm{j})}{\varepsilon}\right|>s \text { for at least two sides }(\mathrm{i}, \mathrm{j}) \text { of } T\right\} .
$$

We claim that we may connect the two opposite sides of $Q_{\rho}^{\nu_{0}}\left(x_{0}\right)$ parallel to $\nu_{0}$ with a path $\left\{T_{i}: i=1, \ldots, M\right\}$ (depending on $\rho$ and $\varepsilon$, but we omit such a dependence for the sake of notational simplicity) consisting of triangles such that $T_{i}$ and $T_{i+1}$ have a common side and $T_{i} \in \mathcal{S}_{\varepsilon}^{s}$ up to a number of indices that is $o(\rho / \varepsilon)$. Indeed, note that for any $T \notin \mathcal{S}_{\varepsilon}^{s}$ we have

$$
\left|\frac{u_{\varepsilon}(i)-u_{\varepsilon}(j)}{\varepsilon}\right| \leq 2 s \text { for every side }(i, j) \text { of } T \text {. }
$$

If no path as above exists then we may construct $c \rho / \varepsilon$ disjoint paths in $L_{\varepsilon} \cap Q_{\rho}^{\nu_{0}}\left(x_{0}\right)$; i.e., sets of indices $\left\{\mathbf{i}_{n}^{j}: n=0, \ldots, M_{j}\right\}$ with $\mathbf{i}_{n}^{j} \in L_{\varepsilon}$ and $\mathbf{i}_{n}^{j}-\mathbf{i}_{n-1}^{j} \in \varepsilon S$, such that

$$
\left\langle i_{0}^{j}-x_{0}, \nu_{0}\right\rangle \leq-\frac{1}{2} \rho+\varepsilon, \quad\left\langle i_{M_{j}}^{j}-x_{0}, \nu_{0}\right\rangle \geq \frac{1}{2} \rho-\varepsilon
$$

and

$$
\left|\frac{u_{\varepsilon}\left(i_{n}^{j}\right)-u_{\varepsilon}\left(i_{n-1}^{j}\right)}{\varepsilon}\right| \leq 2 s \text { for every } n
$$

(for a construction of such paths we refer to the proof of Thm. 4(ii) in [11]). Since $v_{\varepsilon, \rho}-u^{ \pm}\left(x_{0}\right)$ converge to 0 close two opposite sides of $Q^{\nu_{0}}$ it is not restrictive to suppose that

$$
\sum_{j} \varepsilon\left|u^{\varepsilon}\left(\dot{i}_{0}^{j}\right)-u^{-}\left(x_{0}\right)\right|+\sum_{j} \varepsilon\left|u^{\varepsilon}\left(\dot{i}_{M_{j}}^{j}\right)-u^{+}\left(x_{0}\right)\right|=\rho o_{\varepsilon}(1) .
$$

By (6.5) we have

$$
\begin{aligned}
c \rho\left|u^{+}\left(x_{0}\right)-u^{-}\left(x_{0}\right)\right| \leq & \sum_{j} \varepsilon\left(\left|u^{+}\left(x_{0}\right)-u^{\varepsilon}\left(\mathbf{i}_{M_{j}}^{j}\right)\right|\right. \\
& \left.+\left|u^{\varepsilon}\left(\mathbf{i}_{M_{j}}^{j}\right)-u^{\varepsilon}\left(\mathbf{i}_{0}^{j}\right)\right|+\left|u^{\varepsilon}\left(\mathbf{i}_{0}^{j}\right)-u^{-}\left(x_{0}\right)\right|\right) \\
\leq & \rho o_{\varepsilon}(1)+\sum_{j} \sum_{n=1}^{M_{j}} \varepsilon\left|u^{\varepsilon}\left(\mathbf{i}_{n}^{j}\right)-u^{\varepsilon}\left(\mathbf{i}_{n-1}^{j}\right)\right| \\
\leq & \rho o_{\varepsilon}(1)+\sum_{j} \sum_{n=1}^{M_{j}} \varepsilon^{2} 2 s \leq \rho o_{\varepsilon}(1)+\rho^{2} 2 s .
\end{aligned}
$$

Dividing by $\rho$ and letting $\varepsilon$ and $\rho$ tend to 0 we then obtain $\left|u^{+}\left(x_{0}\right)-u^{-}\left(x_{0}\right)\right|=0$, which gives a contradiction. 
We consider the connected set

$$
\mathbf{T}_{\varepsilon}=\bigcup_{i=1}^{M} T_{i} .
$$

Note that by the convergence $v_{\varepsilon, \rho} \rightarrow \widetilde{u}$, for all $\delta>0$ the set $\mathbf{T}_{\varepsilon} \cap Q_{\rho}^{\nu_{0}}\left(x_{0}\right)$ will be contained in the strip $\left\{x:\left|\left\langle x-x_{0}, \nu_{0}\right\rangle\right| \leq \delta \rho\right\}$ for $\varepsilon$ sufficiently small. It is not restrictive to suppose that $Q_{\rho}^{\nu_{0}}\left(x_{0}\right) \cap \partial \mathbf{T}_{\varepsilon}$ is composed exactly of two polygonal chains $P_{-}^{\varepsilon}$ and $P_{+}^{\varepsilon}$. Note that the set

$$
\left\{T_{i}: \partial T_{i} \cap P_{-}^{\varepsilon} \neq \emptyset\right\}
$$

still gives a path with the same properties of $\left\{T_{i}\right\}$. We can therefore suppose that this is our original path.

The contribution of the energy restricted to interactions in $\mathbf{T}_{\varepsilon}$ gives

$$
E_{\varepsilon}\left(u_{\varepsilon}, Q_{\rho}^{\nu_{0}}\left(x_{0}\right) \cap \mathbf{T}_{\varepsilon}\right) \geq J(s) \varphi\left(\nu_{0}\right) \rho(1-\delta)
$$

(see Prop. 4.6). We then deduce that

$$
\#\left\{T \in \mathcal{S}_{\varepsilon}^{s}: T \not \subset \mathbf{T}_{\varepsilon}\right\} \leq c \frac{\rho}{\varepsilon} \delta
$$

and, in addition, there exist $c \frac{\rho}{\varepsilon}$ disjoint polygonal chains joining $P_{-}^{\varepsilon}$ and the side of $Q_{\rho}^{\nu_{0}}\left(x_{0}\right)$ lying on $\{y$ : $\left.\left\langle y-x_{0}, \nu\right\rangle=-\rho / 2\right\}$ such that they do not intersect $\mathcal{S}_{\varepsilon}^{s}$. Since $v_{\varepsilon, \rho} \rightarrow \widetilde{u}$, arguing as in (6.6), we deduce that

$$
\int_{P_{ \pm}^{\varepsilon}}\left|u_{\varepsilon}-u^{ \pm}\left(x_{0}\right)\right| \mathrm{d} \mathcal{H}^{1}=\rho o_{\varepsilon}(1)+o(\rho)
$$

as $\varepsilon \rightarrow 0$.

For fixed $\rho>0$ we may now consider the scaled functions

$$
w_{\varepsilon}^{\rho}(y)=\frac{1}{\rho} u_{\varepsilon}\left(x_{0}+\rho y\right)=\frac{1}{\rho} v_{\varepsilon, \rho}(y) .
$$

We may still argue as in Proposition 4.1 and deduce that $w_{\varepsilon}^{\rho}$ converge to $u^{\rho}(y)=\frac{1}{\rho} u\left(x_{0}+\rho y\right)$ as $\varepsilon \rightarrow 0$. Note that $\nabla u^{\rho}(y)=\nabla u\left(x_{0}+\rho y\right)$.

For fixed $\rho$, we may now consider the functions

$$
\tilde{w}_{\varepsilon}^{\rho}=w_{\varepsilon}^{\rho} \chi_{Q^{\nu_{0}} \backslash \frac{1}{\rho} \mathcal{S}_{\varepsilon}^{s}},
$$

which are now discontinuous at $\partial \mathcal{S}_{\varepsilon}^{s}$. They satisfy

(i) the determinant of $\nabla w_{\varepsilon}^{\rho}$ is equibounded, by the definition of $\mathcal{S}_{\varepsilon}^{s}$;

(ii) $\nabla w_{\varepsilon}^{\rho}$ are equibounded;

(iii) $\mathcal{H}^{1}\left(\partial \mathcal{S}_{\varepsilon}^{s}\right)$ is bounded;

(iv) the measures $\frac{\partial w_{\varepsilon}^{\rho}}{\partial \nu^{\perp}} \mathcal{H}^{1}\left\llcorner\partial \mathcal{S}_{\varepsilon}^{s}\right.$ are equibounded, by (ii) and since $\left|\frac{\partial w_{\varepsilon}^{\rho}}{\partial \nu^{\perp}}\right| \leq 2 s$ by definition. Here and below we simply denote by $\nu$ the normal to a discontinuity set without specifying of which function, which is clear from the context.

By Theorem 5.8 in [3] from (i)-(iv) above and the convergence $\tilde{w}_{\varepsilon}^{\rho} \rightarrow u^{\rho}$ we deduce that the measures $\frac{\partial w_{\varepsilon}^{\rho}}{\partial \nu^{\perp}} \mathcal{H}^{1}\left\llcorner\partial \mathcal{S}_{\varepsilon}^{s}\right.$ converge to $\frac{\partial u^{\rho, \delta}}{\partial \nu^{\perp}} \mathcal{H}^{1}\left\llcorner S_{u^{\rho}}\right.$. We choose the orientation of $\nu^{\perp}$ so that the determinant constraint on $u_{\varepsilon}$ can be rewritten on each line segment of $P_{ \pm}^{\varepsilon}$ as

$$
\left\langle\frac{\partial u_{\varepsilon}}{\partial \nu^{\perp}},\left(u_{\varepsilon}^{+}-u_{\varepsilon}^{-}\right)^{\perp}\right\rangle>0
$$



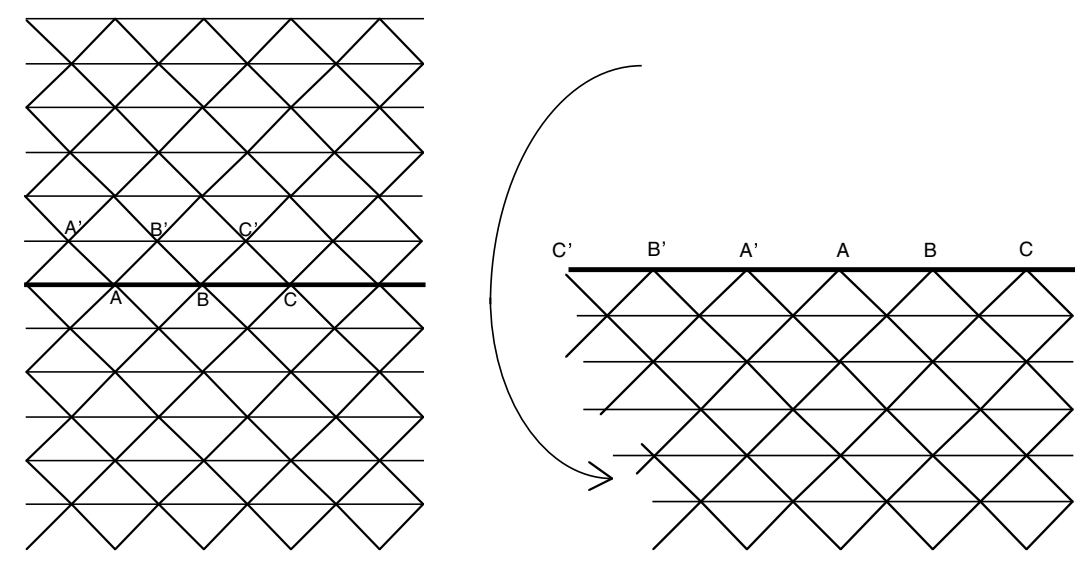

FIgURE 5. Zero-determinant fracture with a planar interface and a 180-degree rotation - reference and deformed microscopic configurations.

where $u_{\varepsilon}^{-}$and $u_{\varepsilon}^{+}$are the values on two vertices of the corresponding triangle, with $u_{\varepsilon}^{-} \in P_{-}^{\varepsilon}$ and $u_{\varepsilon}^{+} \in P_{+}^{\varepsilon}$. We then have, by $(6.9)$

$$
\begin{aligned}
0 & \leq \int_{Q_{\rho}^{\nu_{0}}\left(x_{0}\right) \cap P_{-}^{\varepsilon}}\left\langle\frac{\partial u_{\varepsilon}}{\partial \nu^{\perp}},\left(u_{\varepsilon}^{+}-u_{\varepsilon}^{-}\right)^{\perp}\right\rangle \mathrm{d} \mathcal{H}^{1} \\
& =\int_{Q_{\rho}^{\nu_{0}}\left(x_{0}\right) \cap P_{-}^{\varepsilon}}\left\langle\frac{\partial u_{\varepsilon}}{\partial \nu^{\perp}},\left(u^{+}\left(x_{0}\right)-u^{-}\left(x_{0}\right)\right)^{\perp}\right\rangle \mathrm{d} \mathcal{H}^{1}+\rho o_{\varepsilon}(1)+o(\rho) \\
& =\rho \int_{Q^{\nu_{0}} \cap \frac{1}{\rho}\left(P_{-}^{\varepsilon}-x_{0}\right)}\left\langle\frac{\partial w_{\varepsilon}^{\rho}}{\partial \nu^{\perp}},\left(u^{+}\left(x_{0}\right)-u^{-}\left(x_{0}\right)\right)^{\perp}\right\rangle \mathrm{d} \mathcal{H}^{1}+\rho o_{\varepsilon}(1)+o(\rho) \\
& =\rho \int_{Q^{\nu_{0} \cap S_{u^{\rho}}}}\left\langle\frac{\partial u^{\rho}}{\partial \nu^{\perp}},\left(u^{+}\left(x_{0}\right)-u^{-}\left(x_{0}\right)\right)^{\perp}\right\rangle \mathrm{d} \mathcal{H}^{1}+\rho o_{\varepsilon}(1)+o(\rho) \\
& =\rho\left\langle R^{-} \nu_{0}^{\perp},\left(u^{+}\left(x_{0}\right)-u^{-}\left(x_{0}\right)\right)^{\perp}\right\rangle+\rho o_{\varepsilon}(1)+o(\rho) \\
& =\rho\left\langle R^{-} \nu_{0}, u^{+}\left(x_{0}\right)-u^{-}\left(x_{0}\right)\right\rangle+\rho o_{\varepsilon}(1)+o(\rho) .
\end{aligned}
$$

Dividing by $\rho$ and letting $\varepsilon, \rho \rightarrow 0$ we obtain the first claim in (6.3). The analogous inequality with $R^{+} \nu_{0}$ is obtained in the same way.

Remark 6.2 (Conjecture). We have obtained a weaker necessary condition than the sufficient ones in (5.12). This comes from the fact that in the last argument of the proof we have integrated the positive-determinant constraint on $P_{ \pm}^{\varepsilon}$. We conjecture that this is only a technical issue and indeed those conditions are also necessary in order that the energy density be minimal on $S_{u}$.

\section{Appendix: Positive VS. NON-NEgative Determinant COnstraint}

We have considered a strictly positive microscopic determinant constraint. Strictly positive inequalities are weakened in the limit; however, our choice of not directly considering weak inequalities allows to rule out some additional "unphysical" deformations which would have to be taken into account by directly considering a nonnegative microscopic determinant constraint. This would correspond to allowing microscopic interpolations to "collapse" triangles to line segments on the jump set even though such collapsed triangles cannot be viewed as a limit for the strictly positive determinant case. This may happen in the case of rotations $R^{ \pm} \in \mathcal{S} O(2)$ on both 

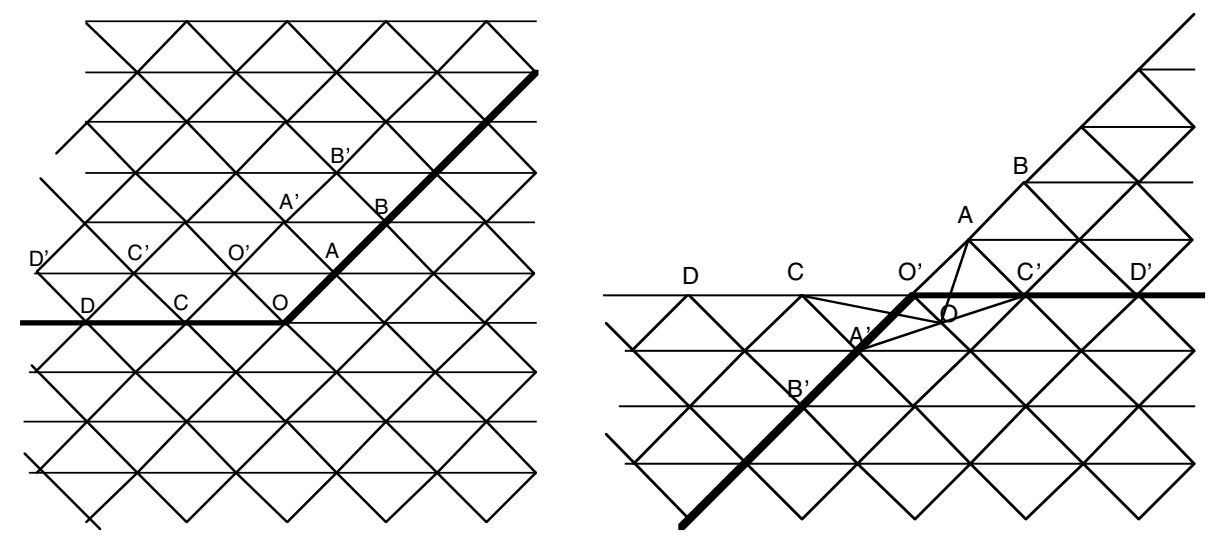

FiguRE 6. Zero-determinant fracture with an angular interface and a 180-degree rotation reference and deformed microscopic configurations.

sides of the interface with $R^{+}=-R^{-}$. In Figure 5 we depict such a microscopic deformation along a single coordinate line, where all points of two rows are aligned. Note that allowing zero-determinant deformations would include such a macroscopic deformation in the set of "minimal interfacial energy", while in our setting the same must be achieved by the introduction of at least one extra layer of atoms, thus doubling the energy.

Another possibility for having $R^{+}=-R^{-}$is with the partition composed of a pair of supplementary angles as in Figure 6. In this case it is possible to "rotate" one of the two angles by $\pi$ with positive or zero determinant on the deformed triangles along the discontinuity set. In both cases, however, we have a macroscopical failure of impenetrability, so we have regarded these cases as degenerate by considering only the strictly-positivedeterminant constraint.

Acknowledgements. We thank the anonymous referees for the valuable comments which greatly improved the presentation of the paper.

\section{REFERENCES}

[1] R. Alicandro, M. Focardi and M.S. Gelli, Finite-difference approximation of energies in fracture mechanics. Ann. Scuola Norm. Sup. Pisa Cl. Sci. 29 (2000) 671-709.

[2] L. Ambrosio and A. Braides, Energies in SBV and variational models in fracture mechanics. In Homogenization and applications to material science. Nice. GAKUTO Internat. Ser. Math. Sci. Appl. 9 (1995) 1-22.

[3] L. Ambrosio, A. Braides and A. Garroni, Special functions with bounded variation and with weakly differentiable traces on the jump set. Nonlin. Differ. Equ. Appl. 5 (1998) 219-243

[4] L. Ambrosio, N. Fusco and D. Pallara, Functions of Bounded Variation and Free Discontinuity Problems. Oxford University Press, Oxford (2000).

[5] B. Bourdin, G.A. Francfort and J.-J. Marigo, The Variational Approach to Fracture. J. Elasticity 91 (2008) 5-148.

[6] A. Braides, $\Gamma$-convergence for Beginners. Oxford University Press, Oxford (2002).

[7] A. Braides, Local minimization, variational evolution and $\Gamma$-convergence. Lect. Notes Math., Springer Verlag, Berlin 2094 (2013).

[8] A. Braides and M.S. Gelli, Limits of discrete systems without convexity hypotheses. Math. Mech. Solids 7 (2002) 41-66.

[9] A. Braides and M.S. Gelli, Asymptotic analysis of microscopic impenetrability constraints for atomistic systems. J. Mech. Phys. Solids 96 (2016) 235-251.

[10] A. Braides, A. Lew and M. Ortiz, Effective cohesive behaviour of layers of interatomic planes. Arch. Ration. Mech. Anal. 180 (2006) 151-182.

[11] A. Braides and A. Piatnitski, Overall properties of a discrete membrane with randomly distributed defects. Arch. Ration. Mech. Anal. 189 (2008) 301-323

[12] A. Braides, M. Solci and E. Vitali, A derivation of linear elastic energies from pair-interaction atomistic systems. Netw. Heterog. Media 2 (2007) 551-567.

[13] A. Braides and L. Truskinovsky, Asymptotic expansions by Gamma-convergence. Cont. Mech. Therm. 20 (2008) 21-62 
[14] A. Chambolle, A. Giacomini and M. Ponsiglione, Piecewise rigidity. J. Funct. Anal. 244 (2007) 134-153.

[15] G. Congedo and I. Tamanini, Problemi di partizioni ottimali con dati illimitati. Atti Accad. Naz. Lincei Cl. Sci. Fis. Mat. Natur. Rend. Lincei Mat. Appl. 4 (1993) 103-108.

[16] G.A. Francfort and J.-J. Marigo, Revisiting brittle fracture as an energy minimization problem. J. Mech. Phys. Solids 46 (1998) 1319-1342.

[17] M. Friedrich, A derivation of linearized Griffith energies from nonlinear models. Arch. Ration. Mech. Anal. 225 (2017) 425-467.

[18] M. Friedrich and B. Schmidt, An atomistic-to-continuum analysis of crystal cleavage in a two-dimensional model problem. J. Nonlin. Sci. 24 (2014) 145-183.

[19] M. Friedrich and B. Schmidt, On a discrete-to-continuum convergence result for a two dimensional brittle material in the small displacement regime. Netw. Heterog. Media 10 (2015) 321-342

[20] M. Friedrich and B. Schmidt, An analysis of crystal cleavage in the passage from atomistic models to continuum theory. Arch. Ration. Mech. Anal. 217 (2015) 263-308.

[21] G. Friesecke and F. Theil, Validity and failure of the Cauchy-Born hypothesis in a two-dimensional mass-spring lattice. J. Nonlin. Sci. 12 (2002) 445-478.

[22] Y.G. Reshetnyak. Liouville's theorem on conformal mappings for minimal regularity as- sumptions. Sib. Math. J. 8 (1967) 69-85.

[23] G. Strang, Linear Algebra and its Applications, 2nd edition, Academic Press, New York (1980).

[24] A. Theil, A proof of crystallization in two dimensions. Commun. Math. Phys. 262 (2006) 209-236.

[25] A. Theil, Surface energies in a two-dimensional mass-spring model for crystals. ESAIM Math. Model. Numer. Anal. 45 (2011) $873-899$.

[26] L. Truskinovsky, Fracture as a phase transition, Contemporary Research in the Mechanics and Mathematics of Materials edited by R.C. Batra and M.F. Beatty. CIMNE, Barcelona (1996) 322-332. 